RESEARCH PAPER RP1699

Part of Journal of Research of the National Bureau of Standards, Volume 36, February 1946

\title{
PHASE EQUILIBRIUM RELATIONS IN A PORTION OF THE SYSTEM Na $\mathrm{Na}_{2} \mathrm{O}-\mathrm{CaO}-\mathrm{Al}_{2} \mathrm{O}_{3}-\mathrm{SiO}_{2}$
}

\author{
By Kenneth T. Greene and R. H. Bogue
}

\section{ABSTRACT}

A study of a portion of the system $\mathrm{Na}$ O-CaO- $\mathrm{Al}_{2} \mathrm{O}_{3}-\mathrm{SiO}_{2}$ has been made as a step in the solution of the problem of the state of combination of $\mathrm{Na}_{2} \mathrm{O}$ in portland cement clinker. Quenching data are given for compositions in the tetrahedron formed by $2 \mathrm{CaO} . \mathrm{SiO}_{2}, \mathrm{CaO}, \mathrm{Na}_{2} \mathrm{O} . \mathrm{Al}_{2} \mathrm{O}_{3}$, and $\mathrm{Al}_{2} \mathrm{O}_{3}$, and phase diagrams are shown for three composition planes through the space model. Five invariant points were located approximately, at one of which $2 \mathrm{CaO} . \mathrm{SiO}_{2}, 3 \mathrm{CaO} . \mathrm{SiO}_{2}, 3 \mathrm{CaO} . \mathrm{Al}_{2} \mathrm{O}_{3}$, and $\mathrm{Na}_{2} \mathrm{O} .8 \mathrm{CaO} \cdot 3 \mathrm{Al}_{2} \mathrm{O}_{3}$ are in equilibrium with liquid. This point, which is not a eutectic, occurs at $1,440^{\circ} \pm 10^{\circ} \mathrm{C}$ and has a composition of 3.5 percent $\mathrm{Na}_{2} \mathrm{O}$, 55.2 percent $\mathrm{CaO}, 31.0$ percent $\mathrm{Al}_{2} \mathrm{O}_{3}$, and 10.3 percent $\mathrm{SiO}_{2}$. Evidence was obtained of the formation, under favorable conditions, of a solid solution between $3 \mathrm{CaO} . \mathrm{Al}_{2} \mathrm{O}_{3}$ and $\mathrm{Na}_{2} \mathrm{O} .8 \mathrm{CaO} .3 \mathrm{Al}_{2} \mathrm{O}_{3}$ at temperatures below that of liquid formation. Present information indicates that $\mathrm{Na}_{2} \mathrm{O}$ may be present in clinker in several possible forms: (1) in glass, (2) as $\mathrm{Na}_{2} \mathrm{O} .8 \mathrm{CaO} .3 \mathrm{Al}_{2} \mathrm{O}_{3}$ or a solid solution of this compound and $3 \mathrm{CaO} . \mathrm{Al}_{2} \mathrm{O}_{3}$, (3) in solid solution in $2 \mathrm{CaO} . \mathrm{SiO}_{2},(4)$ as inclusions of a soda-bearing phase in $\beta$-2CaO. $\mathrm{SiO}_{2}$ produced by ex-solution on inversion from $\alpha-2 \mathrm{CaO} . \mathrm{SiO}_{2}$.

\section{CONTENTS}

II. Experimental method

III. Phase equilibria

1. General discussion

2. The plane $2 \mathrm{CaO} . \mathrm{SiO}_{2}-\mathrm{CaO}-\left(\mathrm{Na}_{2} \mathrm{O}+6 \mathrm{Al}_{2} \mathrm{O}_{3}\right) \ldots \ldots$

3. The plane $2 \mathrm{CaO} . \mathrm{SiO}_{2}-\mathrm{CaO}-\left(\mathrm{Na}_{2} \mathrm{O}+3 \mathrm{Al}_{2} \mathrm{O}_{3}\right) \ldots \ldots$

4. The system $2 \mathrm{CaO} . \mathrm{SiO}_{2}-\mathrm{CaO}-\mathrm{Na}_{2} \mathrm{O} \cdot \mathrm{Al}_{2} \mathrm{O}_{3} \ldots$

5. Additional compositions, located between the $2 \mathrm{CaO} . \mathrm{SiO}_{2}-\mathrm{CaO}-$ $\left(\mathrm{Na}_{2} \mathrm{O}+6 \mathrm{Al}_{2} \mathrm{O}_{3}\right)$ and $2 \mathrm{CaO} \cdot \mathrm{SiO}_{2}-\mathrm{CaO}-\left(\mathrm{Na}_{2} \mathrm{O}+3 \mathrm{Al}_{2} \mathrm{O}_{3}\right)$ planes 201

202

1. Crystallization in the system

2. Solid solutions_._.

3. Applications to portland cement clinker

V. Summary

VI. References_... 207

\section{INTRODUCTION}

In order to understand the state of chemical combination of the various component oxides of portland cement, it is necessary first to study the interrelations of these oxides as systems of few components, gradually extending the scope of the investigations to include other oxides and, consequently, more complicated systems, as more and more data become available. The phase equilibrium relations of the major oxides of portland cement, $\mathrm{CaO}, \mathrm{SiO}_{2}, \mathrm{Al}_{2} \mathrm{O}_{3}, \mathrm{Fe}_{2} \mathrm{O}_{3}$, and $\mathrm{MgO}$ have been rather thoroughly determined and the results are well known. However, it is only within the last few years that a systematic 
attempt has been made to study the effect of such minor components as $\mathrm{SO}_{3}, \mathrm{~K}_{2} \mathrm{O}, \mathrm{Na}_{2} \mathrm{O}, \mathrm{TiO}_{2}$, etc., upon the constitution of portland cement clinker. This paper represents part of an investigation undertaken to determine the manner in which one of these minor components, $\mathrm{Na}_{2} \mathrm{O}$, combines with the other oxides of clinker.

The quaternary system $\mathrm{Na}_{2} \mathrm{O}-\mathrm{CaO}-\mathrm{Al}_{2} \mathrm{O}_{3}-\mathrm{SiO}_{2}$ is one of several which have a direct bearing on the effect of $\mathrm{Na}_{2} \mathrm{O}$ on the phase composition of portland cement clinker. It is probably the most important of these systems, since it contains the three oxides which are usually in greatest abundance, $\mathrm{CaO}, \mathrm{SiO}_{2}$, and $\mathrm{Al}_{2} \mathrm{O}_{3}$. Ferric oxide is the only major component of clinker that is likely to have any radical effect on the phase relations as determined in the $\mathrm{Na} 2 \mathrm{O}-\mathrm{CaO}-\mathrm{Al}_{2} \mathrm{O}_{3}-\mathrm{SiO}_{2}$ system, since $\mathrm{MgO}$ has been shown to exist in clinker essentially uncombined $[1,2,3]$.

One of the four ternary systems bounding the four-component system, which has been determined, is the system $\mathrm{CaO}-\mathrm{Al}_{2} \mathrm{O}_{3}-\mathrm{SiO}_{2}$ originally studied by Rankin and Wright [4]. Portions of the other three ternary systems, each of which contains $\mathrm{Na}_{2} \mathrm{O}$ as a component, have also been studied. However, the only earlier work which has a direct application to portland cement compositions is that of Brownmiller and $\mathrm{Bogue}$ on the system $\mathrm{Na}_{2} \mathrm{O}-\mathrm{CaO}-\mathrm{Al}_{2} \mathrm{O}_{3}$ [5]. They investigated the region of the smaller ternary system $\mathrm{Na}_{2} \mathrm{O} \cdot \mathrm{Al}_{2} \mathrm{O}_{3}-\mathrm{CaO}-\mathrm{Al}_{2} \mathrm{O}_{3}$. The most important result of this work was the discovery of a compound having the formula, $\mathrm{Na}_{2} \mathrm{O} .8 \mathrm{CaO} .3 \mathrm{Al}_{2} \mathrm{O}_{3}$, and being capable of existing in equilibrium with $3 \mathrm{CaO} \cdot \mathrm{Al}_{2} \mathrm{O}_{3}$. As $3 \mathrm{CaO} \cdot \mathrm{Al}_{2} \mathrm{O}_{3}$ is a constituent of portland cement clinker, it appeared that this compound had a possibility of existence in clinkers containing $\mathrm{Na}_{2} \mathrm{O}$. However, the behavior of $\mathrm{Na}_{2} \mathrm{O} .8 \mathrm{CaO} .3 \mathrm{Al}_{2} \mathrm{O}_{3}$ in the presence of the calcium silicates, $3 \mathrm{CaO} . \mathrm{SiO}_{2}$, and $2 \mathrm{CaO} . \mathrm{SiO}_{2}$, had not yet been determined.

Certain limited portions of the quaternary system have also been investigated, but the ranges of composition of these are mostly far removed from the region of interest from the standpoint of portland cement. Consequently, such data will not be considered here. A previous paper from this laboratory by one of the present authors [6] dealt with solid solutions which the compound $2 \mathrm{CaO} \cdot \mathrm{SiO}_{2}$ may form under suitable conditions in the system $\mathrm{Na}_{2} \mathrm{O}-\mathrm{CaO}-\mathrm{Al}_{2} \mathrm{O}_{3}-\mathrm{SiO}_{2}$, as well as in the system $\mathrm{Na}_{2} \mathrm{O}-\mathrm{CaO}-\mathrm{Fe}_{2} \mathrm{O}_{3}-\mathrm{SiO}_{2}$. It was shown that these solid solution phases have a hexagonal crystal structure and that this structure very likely is fundamentally that of the $\alpha$ form of $2 \mathrm{CaO} . \mathrm{SiO}_{2}$. The relation of these phases to the $2 \mathrm{CaO} \cdot \mathrm{SiO}_{2}$ of commercial portland cement clinker was discussed at some length in this paper.

To determine whether $\mathrm{Na}_{2} \mathrm{O} .8 \mathrm{CaO} .3 \mathrm{Al}_{2} \mathrm{O}_{3}$ is the compound of $\mathrm{Na}_{2} \mathrm{O}$ which is stable in mixtures of $\mathrm{Na}_{2} \mathrm{O}, \mathrm{CaO}, \mathrm{Al}_{2} \mathrm{O}_{3}$, and $\mathrm{SiO}_{2}$ in which these oxides are present in the same proportions as in portland cement, it was necessary to study in some detail the phase equilibria in that part of the quaternary system which contains the compounds $3 \mathrm{CaO}$. $\mathrm{SiO}_{2}, \quad 2 \mathrm{CaO} . \mathrm{SiO}_{2}, 3 \mathrm{CaO} \cdot \mathrm{Al}_{2} \mathrm{O}_{3}$, and $\mathrm{Na}_{2} \mathrm{O} .8 \mathrm{CaO} .3 \mathrm{Al}_{2} \mathrm{O}_{3}$. For this purpose the portion of the system consisting of compositions which may be made up of the four compounds $2 \mathrm{CaO} \cdot \mathrm{SiO}_{2}, \mathrm{CaO}, \mathrm{Na}_{2} \mathrm{O} \cdot \mathrm{Al}_{2} \mathrm{O}_{3}$, and $\mathrm{Al}_{2} \mathrm{O}_{3}$ wais chosen. When a regular tetrahedron is used to represent the larger oxide system $\mathrm{Na}_{2} \mathrm{O}-\mathrm{CaO}-\mathrm{Al}_{2} \mathrm{O}_{3}-\mathrm{SiO}_{2}$, the four compounds $2 \mathrm{CaO} . \mathrm{SiO}_{2}, \mathrm{CaO}, \mathrm{Na}_{2} \mathrm{O} \cdot \mathrm{Al}_{2} \mathrm{O}_{3}$, and $\mathrm{Al}_{2} \mathrm{O}_{3}$ are at the apexes of a tetrahedron of unequal dimensions which occupies a space within the larger tetrahedron. 
Various methods are possible for representing the space relations in the model of a four-component system. The ultimate purpose is to define the limits of the regions of primary crystallization of the various phases, called primary phase volumes, and to determine the temperature relations within the primary phase volumes and particularly on the surfaces, along the lines, and at the points where two, three, or four primary phase volumes adjoin one another. It is customary to select various lines or planes through the space model and to determine the manner in which these intersect the primary phase volumes. The lines or planes may represent binary or ternary systems within the quaternary system, they may represent joins which are not true binary or ternary systems, or they may depict planes having arbitrary compositional relationships. An example of the latter type is a series of planes parallel to one face of the tetrahedron, each plane containing a certain constant percentage of the fourth component. Lea and Parker [7] and Schairer [8] discuss in detail methods of studying quaternary systems, and give diagrams to illustrate the manner in which the data may be presented.

In the present investigation, the phase equilibrium relations of three composition planes through the tetrahedral space model have been studied, as well as a number of extra compositions situated between the planes. Each plane, which is triangular in shape, has the compounds $2 \mathrm{CaO} . \mathrm{SiO}_{2}$ and $\mathrm{CaO}$ at two of its apexes. The third apex represents a mixture of $\mathrm{Na}_{2} \mathrm{O}$ and $\mathrm{Al}_{2} \mathrm{O}_{3}$ in a certain definite proportion. All compositions in the same plane therefore contain $\mathrm{Na}_{2} \mathrm{O}$ and $\mathrm{Al}_{2} \mathrm{O}_{3}$ in a constant molar ratio. One of the planes corresponds to one side of the smaller tetrahedron $2 \mathrm{CaO} . \mathrm{SiO}_{2}-\mathrm{CaO}-$ $\mathrm{Na}_{2} \mathrm{O} \cdot \mathrm{Al}_{2} \mathrm{O}_{3}-\mathrm{Al}_{2} \mathrm{O}_{3}$, whereas the other two occupy positions within it. They may be represented as follows:

$$
\begin{aligned}
& 2 \mathrm{CaO} \cdot \mathrm{SiO}_{2}-\mathrm{CaO}-\left(\mathrm{Na}_{2} \mathrm{O}+6 \mathrm{Al}_{2} \mathrm{O}_{3}\right) \\
& 2 \mathrm{CaO} \cdot \mathrm{SiO}_{2}-\mathrm{CaO}-\left(\mathrm{Na}_{2} \mathrm{O}+3 \mathrm{Al}_{2} \mathrm{O}_{3}\right) \\
& \text { 2CaO.SiO }{ }_{2}-\mathrm{CaO}-\mathrm{Na}_{2} \mathrm{O} \cdot \mathrm{Al}_{2} \mathrm{O}_{3}
\end{aligned}
$$

The planes are arranged in order of increasing proportion of $\mathrm{Na}_{2} \mathrm{O}$.

Each of the first two planes is a section through the quaternary system, in which one of the three components is an arbitrary mixture of $\mathrm{Na}_{2} \mathrm{O}$ and $\mathrm{Al}_{2} \mathrm{O}_{3}$. In the third plane, however, the corresponding component is the definite chemical compound, sodium aluminate. Figure 1 shows the relation of these planes and of the smaller tetrahedron $2 \mathrm{CaO} . \mathrm{SiO}_{2}-\mathrm{CaO}-\mathrm{Na}_{2} \mathrm{O} \cdot \mathrm{Al}_{2} \mathrm{O}_{3}-\mathrm{Al}_{2} \mathrm{O}_{3}$ to the tetrahedron representing the complete $\mathrm{Na}_{2} \mathrm{O}-\mathrm{CaO}-\mathrm{Al}_{2} \mathrm{O}_{3}-\mathrm{SiO}_{2}$ system.

\section{EXPERIMENTAL METHOD}

The quenching method of thermal study was used throughout this investigation. The procedure followed was in general similar to that described in previous publications of this laboratory pertaining to alkali systems $[5,9,10]$. In certain cases minor changes were made, as dictated by the conditions encountered.

As in the earlier work with alkalies, volatilization was a problem, particularly in compositions having a relatively high percentage of $\mathrm{Na}_{2} \mathrm{O}$. For this reason most of the mixtures were given no preliminary heat treatment prior to making the quenching determinations, and the time held at constant temperature was kept as short as possible 
consistent with the attainment of equilibrium. By this means the volatilization of $\mathrm{Na}_{2} \mathrm{O}$ was kept at a minimum, and in the great majority of cases it was not found necessary to add an excess of soda to compensate for this loss.

To test the extent of loss of $\mathrm{Na}_{2} \mathrm{O}$ by volatilization, and also the accuracy of proportioning of the raw materials, several representative mixes were analyzed chemically for $\mathrm{Na}_{2} \mathrm{O}$ after heating for one-half hour at temperatures near those of the quenching experiments. The samples analyzed cover a rather large range of $\mathrm{Na}_{2} \mathrm{O}$ percentages, and the results indicate that, with the exception of those relatively

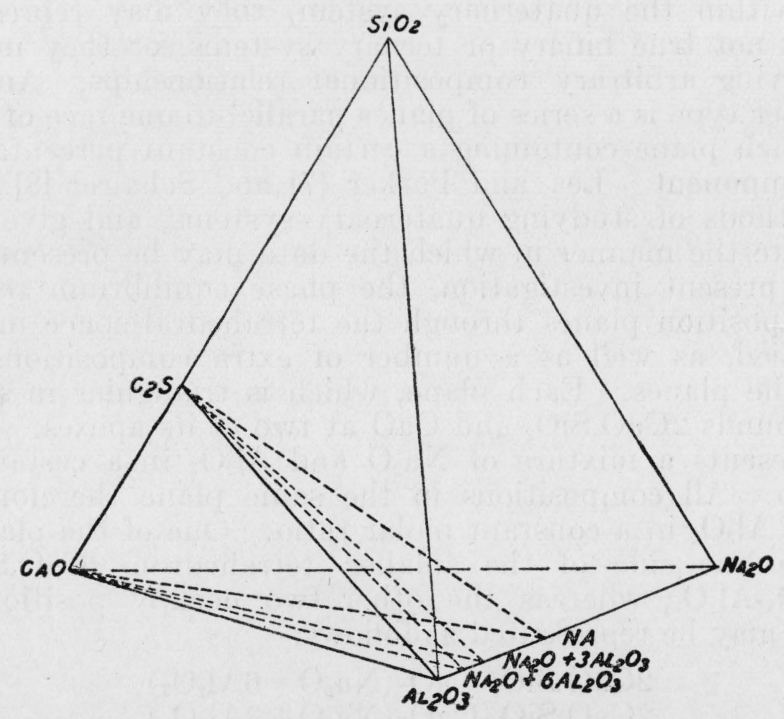

Figure 1.-Tetrahedron $2 \mathrm{CaO} \cdot \mathrm{SiO}_{2}-\mathrm{CaO}-\mathrm{Na}_{2} \mathrm{O} \cdot \mathrm{Al}_{2} \mathrm{O}_{3}-\mathrm{Al}_{2} \mathrm{O}_{3}$ in relation to system $\mathrm{Na}_{2} \mathrm{O}-\mathrm{CaO}-\mathrm{Al}_{2} \mathrm{O}_{3}-\mathrm{SiO}_{2}$.

high in $\mathrm{Na}_{2} \mathrm{O}$, the volatilization is negligible and the amounts of $\mathrm{Na}_{2} \mathrm{O}$ are close to those desired. The data are tabulated below.

$\begin{array}{ccc}\text { Mix number } & \begin{array}{c}\mathrm{Na}_{2} \mathrm{O} \text { desired } \\ 19\end{array} & \begin{array}{c}\mathrm{Na}_{2} \mathrm{O} \text { found } \\ \%\end{array} \\ 24 & 11.34 & 10.99 \\ 60 & 3.78 & 3.79 \\ 101 & 1.69 & 1.63 \\ 204 & 5.90 & 5.89 \\ & 3.88 & 3.97\end{array}$

The omission of preliminary heatings and grindings of the samples used in the quenching furnace made it essential that the raw materials be very thoroughly mixed the first time. This was accomplished by prolonged grinding together of the ingredients in an agate mortar. The distribution of solid phases in the quenched charges, as observed microscopically, indicated that satisfactory mixing had been obtained.

The presence of vapors of $\mathrm{Na}_{2} \mathrm{O}$ in the quenching furnace made it necessary to recalibrate the thermocouple frequently. This was done at times against a standard couple, but more often checks were made against the known melting points of the pure compounds $\mathrm{CaO} . \mathrm{MgO}$.$2 \mathrm{SiO}_{2}\left(1,391.5^{\circ} \mathrm{C}\right)$ and $\mathrm{BaO} .2 \mathrm{SiO}_{2}\left(1,418^{\circ} \mathrm{C}\right)$. 


\section{PHASE EQUILIBRIA}

\section{GENERAL DISCUSSION}

The data on the phase equilibrium relations, as determined by the quenching procedure, are presented in tables $1,2,3$, and 4 . These contain those critical determinations that furnish information necessary to the interpretation of the system. However, they represent only a fraction of the total number of quenching experiments performed. In order to reduce the length of the tables, many data that do not contribute essential information have been omitted. For example, in cases where it was desired to show only which crystalline phase is primary, the results of only one quench are given. Near invariant points or where the liquidus temperatures were determined, data on more than one quench are recorded. In the tables and diagrams the following abbreviations are used:

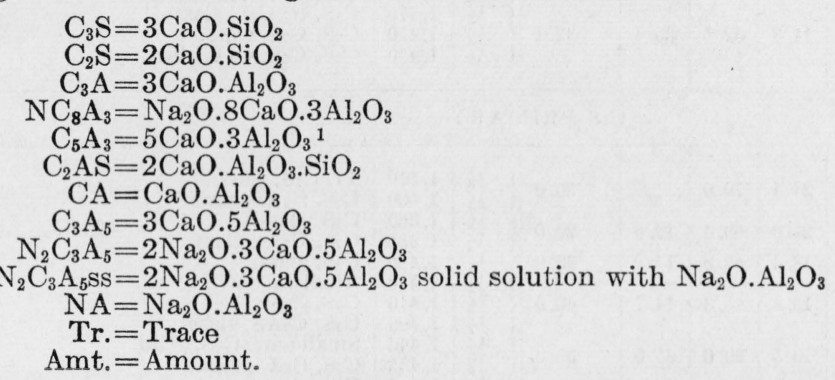

TABLE 1.-Quenching data for compositions in the $2 \mathrm{CaO} \cdot \mathrm{SiO}_{2}-\mathrm{CaO}-\left(\mathrm{Na}_{2} \mathrm{O}+6 \mathrm{Al}_{2} \mathrm{O}_{3}\right)$ plane

\begin{tabular}{|c|c|c|c|c|c|c|c|c|c|c|}
\hline \multirow{3}{*}{ No. } & \multicolumn{7}{|c|}{ Composition } & \multirow{3}{*}{ Time } & \multirow{3}{*}{$\begin{array}{l}\text { Tem- } \\
\text { pera- } \\
\text { ture }\end{array}$} & \multirow{3}{*}{ Phases present } \\
\hline & \multicolumn{4}{|c|}{ Oxides } & \multicolumn{3}{|c|}{ Components } & & & \\
\hline & $\mathrm{Na}_{2} \mathrm{O}$ & $\mathrm{CaO}$ & $\mathrm{Al}_{2} \mathrm{O}_{3}$ & $\mathrm{SiO}_{2}$ & $\mathrm{C}_{2} \mathrm{~S}$ & $\mathrm{CaO}$ & $\begin{array}{c}\left(\mathrm{Na}_{2} \mathrm{O}-\right. \\
+6 \mathrm{Al}_{2-} \\
\left.\mathrm{O}_{3}\right)\end{array}$ & & & \\
\hline
\end{tabular}

CaO PRIMARY

\begin{tabular}{|c|c|c|c|c|c|c|c|c|c|c|}
\hline & $\%$ & $\%$ & $\%$ & $\%$ & $\%$ & $\%$ & $\%$ & $h r$ & ${ }^{\circ} \mathrm{C}$ & \\
\hline 191 & 4. 1 & 55.0 & 40.9 & $-\ldots$ & $-\ldots$ & 55.0 & 45.0 & $\left\{\begin{array}{l}1 / 3 \\
1 / 2\end{array}\right.$ & $\begin{array}{l}1,530 \\
1,520\end{array}$ & $\begin{array}{l}\mathrm{CaO} \text {, glass. } \\
\mathrm{C}_{3} \mathrm{~A} \text {, tr. CaO, glass. }\end{array}$ \\
\hline 192 & 3. 7 & 56.0 & 36.8 & 3.5 & 10.0 & 49.5 & 40.5 & & $\begin{array}{l}1,520 \\
1,515\end{array}$ & $\begin{array}{l}\mathrm{CaO} \text {, glass. } \\
\mathrm{C}_{3} \mathrm{~A}, \mathrm{CaO} \text {, glass. }\end{array}$ \\
\hline 193 & 3. 3 & 57.0 & 32.7 & 7.0 & 20.0 & 44. 0 & 36.0 & $\left\{\begin{array}{l}1 / 4 \\
1 / 4\end{array}\right.$ & $\begin{array}{l}1,485 \\
1,475\end{array}$ & $\begin{array}{l}\mathrm{CaO} \text {, glass. } \\
\mathrm{CaO}, \mathrm{C}_{3} \mathrm{~A} \text {, tr. } \mathrm{NC}_{8} \mathrm{~A}_{3} \text {, glass. }\end{array}$ \\
\hline 194 & 2.9 & 58.0 & 28.6 & 10.5 & 30.0 & 38.5 & 31.5 & $\left\{\begin{array}{l}1 / 6 \\
1 / 3\end{array}\right.$ & $\begin{array}{l}1,510 \\
1,480\end{array}$ & $\begin{array}{l}\mathrm{Tr} . \mathrm{CaO} \text {, tr. } \mathrm{C}_{3} \mathrm{~S} \text {, glass. } \\
\mathrm{CaO}, \mathrm{C}_{3} \mathrm{~S} \text {, glass. }\end{array}$ \\
\hline
\end{tabular}

1 That the results of this investigation might be compared more readily with the literature on the $\mathrm{CaO}$ $\mathrm{Al}_{2} \mathrm{O}_{3}-\mathrm{SiO}_{2}$ system, the formula $5 \mathrm{CaO} .3 \mathrm{Al}_{2} \mathrm{O}_{3}$ is used throughout the paper, although it is recognized that strong evidence has been presented by various authors indicating that the correct formula is $12 \mathrm{CaO}_{2} \mathrm{Al}_{2} \mathrm{O}_{8}$ $[18,19,20,21]$. 
TABLE 1.-Quenching data for compositions in the $2 \mathrm{CaO} \cdot \mathrm{SiO}_{2}-\mathrm{CaO}-\left(\mathrm{Na}_{2} \mathrm{O}+6 \mathrm{Al}_{2} \mathrm{O}_{3}\right)$ plane-Continued

\begin{tabular}{|c|c|c|c|c|c|c|c|c|c|c|}
\hline \multirow{3}{*}{ No. } & \multicolumn{7}{|c|}{ Composition } & \multirow{3}{*}{ Time } & \multirow{3}{*}{$\begin{array}{l}\text { Tem- } \\
\text { pera- } \\
\text { ture }\end{array}$} & \multirow{3}{*}{ Phases present } \\
\hline & \multicolumn{4}{|c|}{ Oxides } & \multicolumn{3}{|c|}{ Components } & & & \\
\hline & $\mathrm{Na}_{2} \mathrm{O}$ & $\mathrm{CaO}$ & $\mathrm{Al}_{2} \mathrm{O}_{3}$ & $\mathrm{SiO}_{2}$ & $\mathrm{C}_{2} \mathrm{~S}$ & $\mathrm{CaO}$ & $\begin{array}{c}\left(\mathrm{Na}_{2} \mathrm{O}-\right. \\
+6 \mathrm{Al}_{2}- \\
\left.\mathrm{O}_{3}\right)\end{array}$ & & & \\
\hline
\end{tabular}

\section{$\mathrm{C}_{3} \mathrm{~S}$ PRIMARY}

\begin{tabular}{|c|c|c|c|c|c|c|c|c|c|c|}
\hline & $\%$ & $\%$ & $\%$ & $\%$ & $\%$ & $\%$ & $\%$ & $h r$ & ${ }^{\circ} \mathrm{C}$ & \\
\hline 196 & 2.7 & 58.5 & 26.6 & 12.2 & 40.0 & 33.0 & 27. 0 & $\left\{\begin{array}{l}1 / 3 \\
1 / 2\end{array}\right.$ & $\begin{array}{l}1,525 \\
1,485\end{array}$ & $\begin{array}{l}\mathrm{C}_{3} \mathrm{~S}, \text { tr. } \mathrm{CaO} \text {, glass. } \\
\mathrm{C}_{3} \mathrm{~S}, \mathrm{CaO} \text {, glass. }\end{array}$ \\
\hline 197 & 3.0 & 56.3 & 30.2 & 10.5 & 30.0 & 36.8 & 33.2 & $\begin{array}{l}1 / 2 \\
1 / 2 \\
1 / 2\end{array}$ & $\begin{array}{l}1,475 \\
1,460 \\
1,450\end{array}$ & $\begin{array}{l}\text { Small amt. } \mathrm{C}_{3} \mathrm{~S} \text {, tr. } \mathrm{CaO} \text {, glass. } \\
\mathrm{C}_{3} \mathrm{~S} \text {, tr. } \mathrm{CaO} \text {, tr. } \mathrm{C}_{2} \mathrm{~S} \text {, glass. } \\
\mathrm{C}_{3} \mathrm{~S}, \mathrm{C}_{2} \mathrm{~S}, \mathrm{C}_{3} \mathrm{~A} \text {, glass. }\end{array}$ \\
\hline 199 & 3.0 & 56.6 & 29.1 & 11. 3 & 32.5 & 35.4 & 32.1 & $\left\{\begin{array}{l}1 / 3 \\
1 / 3 \\
1 / 2\end{array}\right.$ & $\begin{array}{l}1,475 \\
1,470 \\
1,450\end{array}$ & $\begin{array}{l}\mathrm{C}_{2} \mathrm{~S}, \mathrm{tr} . \mathrm{C}_{2} \mathrm{~S}, \text { glass. } \\
\mathrm{C}_{3} \mathrm{~S}, \mathrm{C}_{2} \mathrm{~S}, \text { glass. } \\
\mathrm{C}_{3} \mathrm{~S}, \mathrm{C}_{2} \mathrm{~S}, \mathrm{C}_{3} \mathrm{~A} \text {, glass. }\end{array}$ \\
\hline
\end{tabular}

$\mathrm{C}_{2} \mathrm{~S}$ PRIMARY

\begin{tabular}{|c|c|c|c|c|c|c|c|c|c|c|}
\hline 120 & 2.8 & 45.6 & 27.2 & 24.4 & 70.0 & 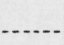 & 30.0 & $\begin{array}{l}1 / 3 \\
1 / 3\end{array}$ & $\begin{array}{l}1,510 \\
1,500\end{array}$ & $\begin{array}{l}\mathrm{Tr} . \mathrm{C}_{2} \mathrm{~S} \text {, glass. } \\
\mathrm{C}_{2} \mathrm{~S}, \mathrm{C}_{2} \mathrm{AS} \text {, glass. }\end{array}$ \\
\hline 132 & 1.8 & 59.0 & 18.2 & 21.0 & 60.4 & 19.6 & 20.0 & $\begin{array}{l}1 / 2 \\
1 / 2\end{array}$ & $\begin{array}{l}1,360 \\
1,355\end{array}$ & $\begin{array}{l}\mathrm{C}_{2} \mathrm{~S} \text {, glass. } \\
\mathrm{C}_{2} \mathrm{~S}, \mathrm{C}_{5} \mathrm{~A}_{3}, \text { glass. }\end{array}$ \\
\hline 133 & 2.8 & 51.6 & 27.2 & 18.4 & 52.8 & 17.2 & 30.0 & $1 / 3$ & 1,450 & $\mathrm{C}_{2} \mathrm{~S}$, glass. \\
\hline 134 & 3.7 & 44. 2 & 36.3 & 15.8 & 45.3 & 14. 7 & 40.0 & $\begin{array}{l}21 / 3 \\
1 / 2\end{array}$ & $\begin{array}{l}1,410 \\
1,400\end{array}$ & $\begin{array}{l}\mathrm{C}_{2} \mathrm{~S} \text {, glass. } \\
\mathrm{C}_{2} \mathrm{~S}, \mathrm{C}_{2} \mathrm{AS} \text {, glass. }\end{array}$ \\
\hline 143 & 3.2 & 54.5 & 31.8 & 10.5 & 30.0 & 35.0 & 35.0 & $\begin{array}{l}1 / 3 \\
1 / 3\end{array}$ & $\begin{array}{l}1,440 \\
1,435\end{array}$ & $\begin{array}{l}\text { Small amt. } \mathrm{C}_{2} \mathrm{~S} \text {, glass. } \\
\mathrm{C}_{2} \mathrm{~S}, \mathrm{C}_{3} \mathrm{~A} \text {, glass. }\end{array}$ \\
\hline 146 & 3.0 & 55.3 & 29.5 & 12.2 & 35.0 & 32.5 & 32.5 & $\begin{array}{l}1 / 3 \\
1 / 2 \\
1 / 3\end{array}$ & $\begin{array}{l}1,440 \\
1,435 \\
1,375\end{array}$ & $\begin{array}{l}\mathrm{C}_{2} \mathrm{~S} \text {, glass. } \\
\mathrm{C}_{2} \mathrm{~S} \text {, small amt. } \mathrm{C}_{3} \mathrm{~A} \text {, glass. } \\
\mathrm{C}_{2} \mathrm{~S} \text {, glass. }\end{array}$ \\
\hline 153 & 3.5 & 51.0 & 35.0 & 10.5 & 30.0 & 31.5 & 38.5 & $\begin{array}{l}1 / 2 \\
1 / 2 \\
1 / 3\end{array}$ & $\begin{array}{l}1,373 \\
1,370 \\
1,360\end{array}$ & $\begin{array}{l}\mathrm{C}_{2} \mathrm{~S}, \mathrm{NC}_{8} \mathrm{~A}_{3} \text {, glass. } \\
\mathrm{C}_{2} \mathrm{~S}, \mathrm{C}_{5} \mathrm{~A}_{3}, \mathrm{NC}_{8} \mathrm{~A}_{3} \text {, glass. } \\
\text { Almost completely crystalline. }\end{array}$ \\
\hline 158 & 3.9 & 47.5 & 38.1 & 10.5 & 30.0 & 28.0 & 42.0 & $\begin{array}{l}1 / 3 \\
1 / 3\end{array}$ & $\begin{array}{l}1,370 \\
1,365\end{array}$ & $\begin{array}{l}\mathrm{C}_{2} \mathrm{~S} \text {, glass. } \\
\mathrm{C}_{2} \mathrm{~S}, \mathrm{C}_{5} \mathrm{~A}_{3} \text {, glass. }\end{array}$ \\
\hline $\begin{array}{l}165 \\
170\end{array}$ & $\begin{array}{l}3.7 \\
3.9\end{array}$ & $\begin{array}{l}50.5 \\
45.5\end{array}$ & $\begin{array}{l}36.2 \\
38.4\end{array}$ & $\begin{array}{r}9.6 \\
12.2\end{array}$ & $\begin{array}{l}27.5 \\
35.0\end{array}$ & $\begin{array}{l}32.6 \\
22.7\end{array}$ & $\begin{array}{l}39.9 \\
42.3\end{array}$ & $1 / 3$ & 1,375 & $\mathrm{C}_{2} \mathrm{~S}$, glass. \\
\hline 184 & 4. 1 & 43.5 & 40.9 & 11.5 & 33.0 & 22.0 & 45.0 & $\begin{array}{l}1 / 3 \\
1 / 2\end{array}$ & $\begin{array}{l}1,350 \\
1,345\end{array}$ & $\begin{array}{l}\text { Tr. } \mathrm{C}_{2} \mathrm{~S} \text {, glass. } \\
\mathrm{C}_{2} \mathrm{~S}, \mathrm{C}_{5} \mathrm{~A}_{3} \text {, glass. }\end{array}$ \\
\hline 195 & 2.5 & 59.1 & 24.5 & 13.9 & 40.0 & 33.0 & 27.0 & $1 / 3$ & 1,525 & $\mathrm{C}_{2} \mathrm{~S}, \mathrm{C}_{3} \mathrm{~S}$, glass. Probably near \\
\hline 198 & 2.8 & 57. 0 & 28.0 & 12.2 & 35.0 & 34.1 & 30.9 & $\begin{array}{l}1 / 4 \\
1 / 2 \\
1 / 2 \\
1 / 2\end{array}$ & $\begin{array}{l}1,475 \\
1,460 \\
1,450 \\
1,445\end{array}$ & $\begin{array}{l}\mathrm{C}_{2} \mathrm{~S}, \text { tr. } \mathrm{C}_{3} \mathrm{~S} \text {, glass. } \\
\mathrm{C}_{2} \mathrm{~S}, \mathrm{C}_{3} \mathrm{~S}, \text { glass. } \\
\mathrm{C}_{2} \mathrm{~S}, \mathrm{C}_{3} \mathrm{~S}, \mathrm{C}_{3} \mathrm{~A} \text {, glass. } \\
\mathrm{C}_{2} \mathrm{~S}, \mathrm{C}_{3} \mathrm{~A} \text {, glass. }\end{array}$ \\
\hline
\end{tabular}

\section{$\mathrm{C}_{3} \mathrm{~A}$ PRIMARY}

\begin{tabular}{|c|c|c|c|c|c|c|c|c|c|c|}
\hline 140 & 4. 6 & 50.0 & 45.4 & 35 & 100 & 50.0 & 50.0 & $1 / 2$ & $\begin{array}{l}1,450 \\
1,505\end{array}$ & $\mathrm{C}_{3} \mathrm{~A}$, glass. \\
\hline 141 & & 51.5 & & 3.5 & 10.0 & & 40.0 & $1 / 3$ & $\begin{array}{l}1,000 \\
1,465\end{array}$ & All glass. \\
\hline 142 & 3.7 & 53.0 & 36.3 & 7.0 & 20.0 & 40.0 & 40.0 & $1 / 2$ & $\begin{array}{l}1,400 \\
1,460 \\
1,450\end{array}$ & $\begin{array}{l}\mathrm{Tr} \text {. } \mathrm{C}_{3} \mathrm{~A} \text {, glass. } \\
\text { All glass. }\end{array}$ \\
\hline 145 & 3.4 & 53.8 & 34.1 & 8.7 & 25.0 & 37.5 & 37.5 & $\begin{array}{l}1 \\
1 / 4 \\
1 / 4\end{array}$ & $\begin{array}{l}1,445 \\
1,430\end{array}$ & $\begin{array}{l}\mathrm{C}_{3} \mathrm{~A} \text {, glass. } \\
\mathrm{C}_{3} \mathrm{~A} \text {, tr. } \mathrm{C}_{2} \mathrm{~S} \text {, glass. }\end{array}$ \\
\hline 147 & 3.3 & 54. 2 & 32.9 & 9.6 & 27.5 & 36.3 & 36. 2 & $\begin{array}{l}1 / 2 \\
1 / 3\end{array}$ & $\begin{array}{l}1,445 \\
1,440\end{array}$ & $\begin{array}{l}\text { All glass. } \\
\mathrm{C}_{3} \mathrm{~A} \text {, tr. } \mathrm{C}_{2} \mathrm{~S} \text {, glass. }\end{array}$ \\
\hline 160 & 4. 3 & 49.3 & 42.9 & 3.5 & 10.0 & 42.7 & 47.3 & $\begin{array}{l}1 / 3 \\
1 / 3\end{array}$ & $\begin{array}{l}1,450 \\
1,440\end{array}$ & $\begin{array}{l}\text { All glass. } \\
\mathrm{C}_{3} \mathrm{~A} \text {, glass. }\end{array}$ \\
\hline 161 & 3. 9 & 51.0 & 38.1 & 7.0 & 20.0 & 38.0 & 42. 0 & $\begin{array}{l}1 / 3 \\
1 / 3\end{array}$ & $\begin{array}{l}1,425 \\
1,410\end{array}$ & $\begin{array}{l}\text { All glass. } \\
\mathrm{C}_{3} \mathrm{~A} \text {, glass. }\end{array}$ \\
\hline 162 & 3.6 & 51. 9 & 35.8 & 8.7 & 25.0 & 35.6 & 39.4 & $\begin{array}{l}1 / 3 \\
1 / 3\end{array}$ & $\begin{array}{l}1,420 \\
1,415\end{array}$ & $\begin{array}{l}\text { All glass. } \\
\mathrm{C}_{3} \mathrm{~A} \text {, tr. } \mathrm{C}_{2} \mathrm{~S} \text {, glass. }\end{array}$ \\
\hline
\end{tabular}


TABLE 1.-Quenching data for compositions in the $2 \mathrm{CaO} \cdot \mathrm{SiO}_{2}-\mathrm{CaO}-\left(\mathrm{Na}_{2} \mathrm{O}+6 \mathrm{Al}_{2} \mathrm{O}_{3}\right)$ plane - Continued

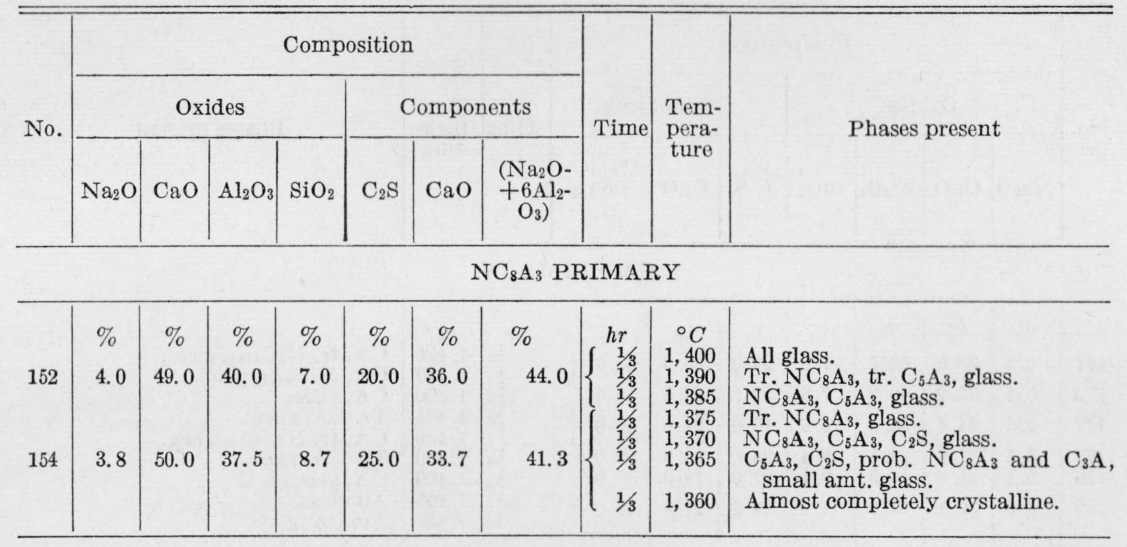

\section{$\mathrm{C}_{5} \mathrm{~A}_{3}$ PRIMARY}

\begin{tabular}{|c|c|c|c|c|c|c|c|c|c|c|}
\hline 150 & 5.1 & 45.0 & 49.9 & ....... & & 45.0 & 55.0 & $\left\{\begin{array}{l}1 / 2 \\
1 / 2\end{array}\right.$ & $\begin{array}{l}1,450 \\
1,445\end{array}$ & $\begin{array}{l}\text { All glass. } \\
\mathrm{C}_{5} \mathrm{~A}_{3} \text {, glass. }\end{array}$ \\
\hline 151 & 4.5 & 47. 0 & 45.0 & 3.5 & 10.0 & 40.5 & 49.5 & $1 / 3$ & $\begin{array}{l}1,425 \\
1,420\end{array}$ & $\begin{array}{l}\text { All glass. } \\
\mathrm{C}_{5} \mathrm{~A}_{3}, \text { glass. }\end{array}$ \\
\hline 156 & 5.0 & 42.5 & 49.0 & 3.5 & 10.0 & 36.0 & 54.0 & $\left\{\begin{array}{l}1 / 3 \\
1 / 3\end{array}\right.$ & $\begin{array}{l}1,440 \\
1,435\end{array}$ & $\begin{array}{l}\text { All glass. } \\
\mathrm{C}_{5} \mathrm{~A}_{3}, \text { glass. }\end{array}$ \\
\hline 157 & 4. 4 & 45.0 & 43.6 & 7.0 & 20.0 & 32.0 & 48.0 & $1 / 3$ & 1,410 & All glass. \\
\hline 159 & 4.1 & 46.3 & 40.9 & 8.7 & 25.0 & 30.0 & 45.0 & $1 / 3$ & 1,390 & Do. \\
\hline 163 & 4. 2 & 47.0 & 41.8 & 7.0 & 20.0 & 34.0 & 46.0 & $\left\{\begin{array}{l}1 / 3 \\
1 / 3\end{array}\right.$ & $\begin{array}{l}1,400 \\
1,395\end{array}$ & $\begin{array}{l}\text { Perhaps tr. } \mathrm{C}_{5} \mathrm{~A}_{3} \text {, glass. } \\
\mathrm{C}_{5} \mathrm{~A}_{3} \text {, glass. }\end{array}$ \\
\hline 164 & 4. 0 & 48.1 & 39.2 & 8.7 & 25.0 & 31.9 & 43.1 & $\left\{\begin{array}{l}1 / 3 \\
1 / 3\end{array}\right.$ & $\begin{array}{l}1,370 \\
1,365\end{array}$ & $\mathrm{C}_{5} \mathrm{~A}_{3}, \mathrm{C}_{2} \mathrm{~S}$, glass. \\
\hline 168 & 4.8 & 41.0 & 47.2 & 7.0 & 20.0 & 28.0 & 52.0 & $\left\{\begin{array}{l}1 / 3 \\
1 / 3\end{array}\right.$ & $\begin{array}{l}1,390 \\
1,380\end{array}$ & $\begin{array}{l}\text { All glass. } \\
\mathrm{C}_{5} \mathrm{~A}_{3} \text {, glass. }\end{array}$ \\
\hline 169 & 4. 2 & 44.0 & 41.3 & 10.5 & 30.0 & 24.5 & 45.5 & $\left\{\begin{array}{l}1 / 3 \\
1 / 3\end{array}\right.$ & $\begin{array}{l}1,375 \\
1,360\end{array}$ & $\begin{array}{l}\text { All glass. } \\
\mathrm{C}_{5} \mathrm{~A}_{3}, \text { glass. }\end{array}$ \\
\hline 175 & 5.1 & 38.7 & 49.9 & 6.3 & 18.0 & 27.0 & 55.0 & $11 / 4$ & $\begin{array}{l}1,400 \\
1,390\end{array}$ & $\begin{array}{l}\text { All glass. } \\
\mathrm{C}_{5} \mathrm{~A}_{3} \text {, perhaps tr. } \mathrm{CA} \text {, glass. }\end{array}$ \\
\hline 181 & 4. 6 & 41.3 & 45.4 & 8.7 & 25.0 & 25.0 & 50.0 & $\left\{\begin{array}{l}1 / 3 \\
1 / 3\end{array}\right.$ & $\begin{array}{l}1,370 \\
1,365\end{array}$ & $\begin{array}{l}\text { All glass. } \\
\mathrm{C}_{5} \mathrm{~A}_{3} \text {, glass. }\end{array}$ \\
\hline 183 & 4. 6 & 40.4 & 45.4 & 9.6 & 27.5 & 22.5 & 50.0 & $\left\{\begin{array}{l}1 / 3 \\
1 / 3\end{array}\right.$ & $\begin{array}{l}1,350 \\
1,345\end{array}$ & $\begin{array}{l}\mathrm{Tr} . \mathrm{C}_{5} \mathrm{~A}_{3}, \text { glass. } \\
\mathrm{C}_{5} \mathrm{~A}_{3} \text {, tr. } \mathrm{C}_{2} \mathrm{AS} \text {, glass. }\end{array}$ \\
\hline
\end{tabular}

$\mathrm{C}_{2} \mathrm{AS}$ PRIMARY

\begin{tabular}{|c|c|c|c|c|c|c|c|c|c|c|}
\hline 122 & 4. 6 & 32.6 & 45.4 & 17.4 & 50.0 & & 50.0 & $\left\{\begin{array}{l}1 / 3 \\
1 / 3\end{array}\right.$ & $\begin{array}{l}1,510 \\
1,505\end{array}$ & All glass. \\
\hline 128 & 4.8 & 30.9 & 47. 7 & 16. 6 & 52.5 & $\ldots$ & 47.5 & $1 / 4$ & 1,500 & $\mathrm{Tr} . \mathrm{C}_{2} \mathrm{AS}$, glass. \\
\hline 135 & 4.6 & 36.9 & 45.4 & 13.1 & 37.7 & 12.3 & 50.0 & $\left\{\begin{array}{l}1 / 3 \\
1 / 3\end{array}\right.$ & $\begin{array}{l}1,475 \\
1,460\end{array}$ & $\begin{array}{l}\text { All glass. } \\
\mathrm{C}_{2} \mathrm{AS} \text {, glass. }\end{array}$ \\
\hline 137 & 5. 1 & 33.2 & 49. 9 & 11.8 & 34.0 & 11.0 & 55.0 & $1 / 3$ & 1,450 & $\mathrm{Tr} . \mathrm{C}_{2} \mathrm{AS}$, glass. \\
\hline 180 & 5.1 & 34.0 & 49.9 & 11.0 & 31.5 & 13.5 & 55. 0 & $1 / 4$ & 1,425 & $\mathrm{C}_{2} \mathrm{AS}$, glass. \\
\hline 182 & 4. 6 & 39.5 & 45.4 & 10.5 & 30.0 & 20.0 & 50.0 & $1 / 3$ & 1,375 & Tr. $\mathrm{C}_{2} \mathrm{AS}$, glass. \\
\hline 185 & 4.1 & 41.6 & 40.9 & 13.4 & 38.5 & 16.5 & 45.0 & $1 / 3$ & 1,395 & $\mathrm{C}_{2} \mathrm{AS}$, glass. \\
\hline 186 & 4.1 & 42.5 & 40.9 & 12.5 & 35.8 & 19.2 & 45.0 & $1 / 3$ & 1,350 & $\mathrm{C}_{2} \mathrm{AS}$, glass. \\
\hline 187 & 4.4 & 41.5 & 43.1 & 11.0 & 31.5 & 21.0 & 47.5 & $1 / 2$ & 1,350 & $\mathrm{C}_{2} \mathrm{AS}$, glass. \\
\hline
\end{tabular}


TABLE 1.-Quenching"data for compositions in the $2 \mathrm{CaO} \cdot \mathrm{SiO}_{2}-\mathrm{CaO}-\left(\mathrm{Na}_{2} \mathrm{O}+6 \mathrm{Al}_{2} \mathrm{O}_{3}\right)$ plane-Continued

\begin{tabular}{|c|c|c|c|c|c|c|c|c|c|c|}
\hline \multirow{3}{*}{ No. } & \multicolumn{7}{|c|}{ Composition } & \multirow{3}{*}{ Time } & \multirow{3}{*}{$\begin{array}{l}\text { Tem- } \\
\text { pera- } \\
\text { ture }\end{array}$} & \multirow{3}{*}{ Phases present } \\
\hline & \multicolumn{4}{|c|}{ Oxides } & \multicolumn{3}{|c|}{ Components } & & & \\
\hline & $\mathrm{Na}_{2} \mathrm{O}$ & $\mathrm{CaO}$ & $\mathrm{Al}_{2} \mathrm{O}_{3}$ & $\mathrm{SiO}_{2}$ & $\mathrm{C}_{2} \mathrm{~S}$ & $\mathrm{CaO}$ & $\begin{array}{c}\left(\mathrm{Na}_{2} \mathrm{O}-\right. \\
+6 \mathrm{Al}_{2-} \\
\left.\mathrm{O}_{3}\right)\end{array}$ & & & \\
\hline
\end{tabular}

CA PRIMARY

\begin{tabular}{|c|c|c|c|c|c|c|c|c|c|c|}
\hline & $\%$ & $\%$ & $\%$ & $\%$ & $\%$ & $\%$ & $\%$ & $h r$ & ${ }^{\circ} \mathrm{C}$ & \\
\hline 171 & 5.5 & 33.0 & 54.5 & 7.0 & 20.0 & 20.0 & 60.0 & $\begin{array}{l}1 / 4 \\
1 / 3\end{array}$ & $\begin{array}{l}1,425 \\
1,410\end{array}$ & $\begin{array}{l}\mathrm{CA}, \mathrm{tr}, \mathrm{N}_{2} \mathrm{C}_{3} \mathrm{~A}_{5} \text {, glass. } \\
\mathrm{CA}, \mathrm{N}_{2} \mathrm{C}_{3} \mathrm{~A}_{5} \text {, glass. }\end{array}$ \\
\hline 172 & 5.1 & 37.2 & 49.9 & 7.8 & 22.5 & 22.5 & 55.0 & $1 / 4$ & 1,400 & $\mathrm{CA}$, glass. \\
\hline 173 & 5.5 & 34.4 & 54.5 & 5.6 & 16. 0 & 24.0 & 60.0 & $\begin{array}{l}1 / 4 \\
1 / 4\end{array}$ & $\begin{array}{l}1,435 \\
1,430\end{array}$ & Tr. CA, glass. \\
\hline $\begin{array}{l}176 \\
179\end{array}$ & $\begin{array}{l}5.5 \\
5.1\end{array}$ & $\begin{array}{l}31.6 \\
35.6\end{array}$ & $\begin{array}{l}54.5 \\
49.9\end{array}$ & $\begin{array}{l}8.4 \\
9.4\end{array}$ & $\begin{array}{l}24.0 \\
27.0\end{array}$ & $\begin{array}{l}16.0 \\
18.0\end{array}$ & $\begin{array}{l}60.0 \\
55.0\end{array}$ & $\begin{array}{l}1 / 4 \\
1 / 3 \\
1 / 3\end{array}$ & $\begin{array}{l}1,450 \\
1,400\end{array}$ & $\begin{array}{l}\mathrm{Tr} . \mathrm{CA} \text {, glass. } \\
\mathrm{CA} \text {, glass. }\end{array}$ \\
\hline 189 & 6.0 & 30.1 & 59.0 & 4. 9 & 14. 0 & 21.0 & 65.0 & $\begin{array}{l}1 / 6 \\
1 / 6\end{array}$ & $\begin{array}{l}1,465 \\
1,455\end{array}$ & $\begin{array}{l}\text { All glass. } \\
\text { Tr. CA, glass. }\end{array}$ \\
\hline
\end{tabular}

$\mathrm{N}_{2} \mathrm{C}_{3} \mathrm{~A}_{5}$ PRIMARY

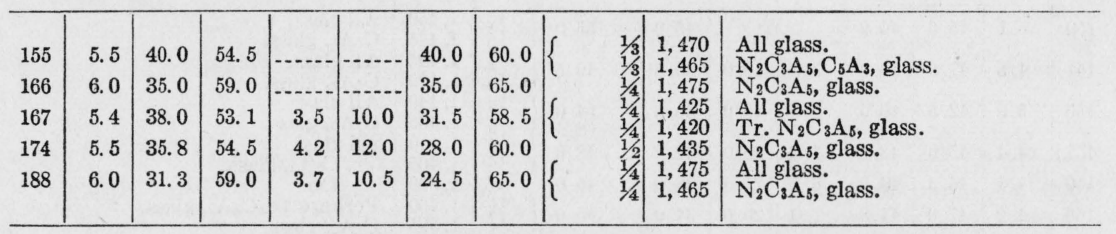

$\mathrm{C}_{3} \mathrm{~A}_{5}$ PRIMARY

\begin{tabular}{|c|c|c|c|c|c|c|c|c|c|c|}
\hline $\begin{array}{l}123 \\
124 \\
126 \\
136 \\
177\end{array}$ & $\begin{array}{l}5.5 \\
6.4 \\
5.1 \\
5.5 \\
5.5\end{array}$ & $\begin{array}{l}26.1 \\
19.5 \\
29.3 \\
29.5 \\
30.2\end{array}$ & $\begin{array}{l}54.5 \\
63.6 \\
49.9 \\
54.5 \\
54.5\end{array}$ & $\begin{array}{r}13.9 \\
10.5 \\
15.7 \\
10.5 \\
9.8\end{array}$ & $\begin{array}{l}40.0 \\
30.0 \\
45.0 \\
30.2 \\
28.0\end{array}$ & $\begin{array}{r}0.8 \\
9.0\end{array}$ & $\begin{array}{l}60.0 \\
70.0 \\
55.0 \\
60.0 \\
60.0\end{array}$ & $\begin{array}{l}1 / 4 \\
116 \\
1 / 3 \\
1 / 3 \\
1 / 4\end{array}$ & $\begin{array}{l}1,550 \\
1,600 \\
1,475 \\
1,460 \\
1,450\end{array}$ & $\begin{array}{c}\mathrm{C}_{3} \mathrm{~A}_{5} \text {, glass. } \\
\text { Do. } \\
\text { Do. } \\
\text { Do. } \\
\text { Do. }\end{array}$ \\
\hline 178 & 5.5 & 30.9 & 54.5 & 9.1 & 26.0 & 14.0 & 60.0 & $\left\{\begin{array}{l}1 / 4 \\
1 / 4\end{array}\right.$ & $\begin{array}{l}1,440 \\
1,435\end{array}$ & $\begin{array}{l}\mathrm{C}_{3} \mathrm{~A}_{5} \text {, tr. } \mathrm{CA} \text {, glass. } \\
\mathrm{C}_{3} \mathrm{~A}_{5}, \mathrm{CA}, \text { glass. }\end{array}$ \\
\hline 190 & 6.0 & 28.9 & 59.0 & 6.1 & 17.5 & 17.5 & 65.0 & $1 / 6$ & 1,460 & $\mathrm{C}_{3} \mathrm{~A}_{3}$, glass. \\
\hline
\end{tabular}

$\mathrm{Al}_{2} \mathrm{O}_{3}$ PRIMARY

\begin{tabular}{|c|c|c|c|c|c|c|c|c|c|c|c|}
\hline 125 & 6.9 & 16. 3 & 68.1 & 8.7 & 25.0 & & 75.0 & $\left\{\begin{array}{ll}1 / 6 \\
1 / 4\end{array} \mid\right.$ & $\begin{array}{l}1,600 \\
1,550\end{array}$ & $\begin{array}{l}\alpha-\mathrm{Al}_{2} \mathrm{O}_{3}, \mathrm{C}_{3} \mathrm{~A}_{5} \text {, glass. } \\
\alpha \text { - } \mathrm{Al}_{2} \mathrm{O}_{3} \text {, more } \mathrm{C}_{3} \mathrm{~A}_{5} \text {, glass. } \\
\text { ably in } \mathrm{Al}_{2} \mathrm{O}_{3} \text { field. }\end{array}$ & Prob- \\
\hline
\end{tabular}


TABLE 2.-Quenching data for compositions in the $2 \mathrm{CaO} . \mathrm{SiO}_{2}-\mathrm{CaO}-\left(\mathrm{Na}_{2} \mathrm{O}+3 \mathrm{Al}_{2} \mathrm{O}_{3}\right)$ plane-Continued

\begin{tabular}{|c|c|c|c|c|c|c|c|c|c|c|}
\hline \multirow{3}{*}{ No. } & \multicolumn{7}{|c|}{ Composition } & \multirow{3}{*}{ Time } & \multirow{3}{*}{$\begin{array}{l}\text { Tem- } \\
\text { pera- } \\
\text { ture }\end{array}$} & \multirow{3}{*}{ Phases present } \\
\hline & \multicolumn{4}{|c|}{ Oxides } & \multicolumn{3}{|c|}{ Components } & & & \\
\hline & $\mathrm{Na}_{2} \mathrm{O}$ & $\mathrm{CaO}$ & $\mathrm{Al}_{2} \mathrm{O}_{3}$ & $\mathrm{SiO}_{2}$ & $\mathrm{C}_{2} \mathrm{~S}$ & $\mathrm{CaO}$ & $\begin{array}{l}\left(\mathrm{Na}_{2} \mathrm{O}+\right. \\
\left.3 \mathrm{Al}_{2} \mathrm{O}_{3}\right)\end{array}$ & & & \\
\hline
\end{tabular}

\section{CaO PRIMARY}

\begin{tabular}{|c|c|c|c|c|c|c|c|c|c|c|}
\hline & $\%$ & $\%$ & $\%$ & $\%$ & $\%$ & $\%$ & $\%$ & $h r$ & ${ }^{\circ} \mathrm{C}$ & \\
\hline 78 & 8.4 & 50.0 & 41. 6 & $-\ldots-1$ & $-\ldots$ & 50.0 & 50.0 & $\begin{array}{l}1 / 2 \\
1 / 3\end{array}$ & $\begin{array}{l}1,510 \\
1,500\end{array}$ & $\begin{array}{l}\mathrm{Small} \text { amt. } \mathrm{CaO} \text {, glass. } \\
\mathrm{NC}_{8} \mathrm{~A}_{3} \text {, glass. }\end{array}$ \\
\hline 79 & 8.4 & 48.3 & 41.6 & 1. 7 & 5.0 & 45.0 & 50.0 & & $\begin{array}{l}1,500 \\
1,490\end{array}$ & $\begin{array}{l}\mathrm{Tr} . \mathrm{CaO}_{2} \text {, glass. } \\
\mathrm{NC}_{8} \mathrm{~A}_{3}, \mathrm{tr} . \mathrm{CaO} \text {, glass. }\end{array}$ \\
\hline 90 & 7.6 & 51.2 & 37.4 & 3.8 & 11.0 & 44.0 & 45.0 & $\begin{array}{l}1 / 3 \\
1 / 3\end{array}$ & $\begin{array}{l}1,500 \\
1,490\end{array}$ & $\begin{array}{l}\mathrm{CaO} \text {, glass. } \\
\mathrm{NC}_{8} \mathrm{~A}_{3}, \mathrm{CaO} \text {, glass. }\end{array}$ \\
\hline 100 & 6.7 & 53.0 & 33.3 & 7. 0 & 20.0 & 40.0 & 40.0 & & $\begin{array}{l}1,485 \\
1,465\end{array}$ & $\begin{array}{l}\mathrm{CaO} \text {, glass. } \\
\mathrm{NC}_{8} \mathrm{~A}_{3}, \mathrm{CaO} \text {, glass. }\end{array}$ \\
\hline 101 & 5. 9 & 54.5 & 29.1 & 10.5 & 30.0 & 35.0 & 35.0 & $\begin{array}{l}1 / 2 \\
1 / 2\end{array}$ & $\begin{array}{l}1,450 \\
1,440\end{array}$ & $\begin{array}{l}\mathrm{CaO} \text {, glass. } \\
\mathrm{NC}_{8} \mathrm{~A}_{3}, \mathrm{CaO}, \text { glass. }\end{array}$ \\
\hline 102 & 5.1 & 56.1 & 24.9 & 13,9 & 40.0 & 30.0 & 30.0 & $\begin{array}{l}1 / 3 \\
1 / 2\end{array}$ & $\begin{array}{l}1,500 \\
1,485 \\
1,450\end{array}$ & CaO, tr. $\mathrm{C}_{3} \mathrm{~S}$, glass. \\
\hline 105 & 5.5 & 55.3 & 27.0 & 12. 2 & 35.0 & 32.5 & 32.5 & $1^{3 / 4}$ & $\begin{array}{l}1,400 \\
1,445 \\
1,440\end{array}$ & 知, tr. $\mathrm{C}_{3} \mathrm{~S}$, tr. $\mathrm{C}_{2} \mathrm{~S}$, glass. \\
\hline 107 & 5.7 & 53.7 & 28.4 & 12. 2 & 35.0 & 30.9 & 34.1 & $\left\{\begin{array}{l}1 / 2 \\
1 / 2\end{array}\right.$ & $\begin{array}{l}1,440 \\
1,435\end{array}$ & $\begin{array}{l}\mathrm{CaO} \text {, tr. } \mathrm{C}_{2} \mathrm{~S} \text {, glass. } \\
\mathrm{C}_{2} \mathrm{~S}, \mathrm{NC}_{8} \mathrm{~A}_{3} \text {, glass. }\end{array}$ \\
\hline
\end{tabular}

C.S PRIMARY

\begin{tabular}{l|l|l|l|l|l|l|l|l|l|l}
\hline 109 & 4.8 & 56.5 & 23.9 & 14.8 & 42.5 & 28.8 & 28.7 & $2 / 3$ & 1,525 & $\mathrm{C}_{3} \mathrm{~S}, \mathrm{C}_{2} \mathrm{~S}$, glass. Near boundary. \\
\hline
\end{tabular}

\section{$\mathrm{C}_{2} \mathrm{~S}$ PRIMARY}

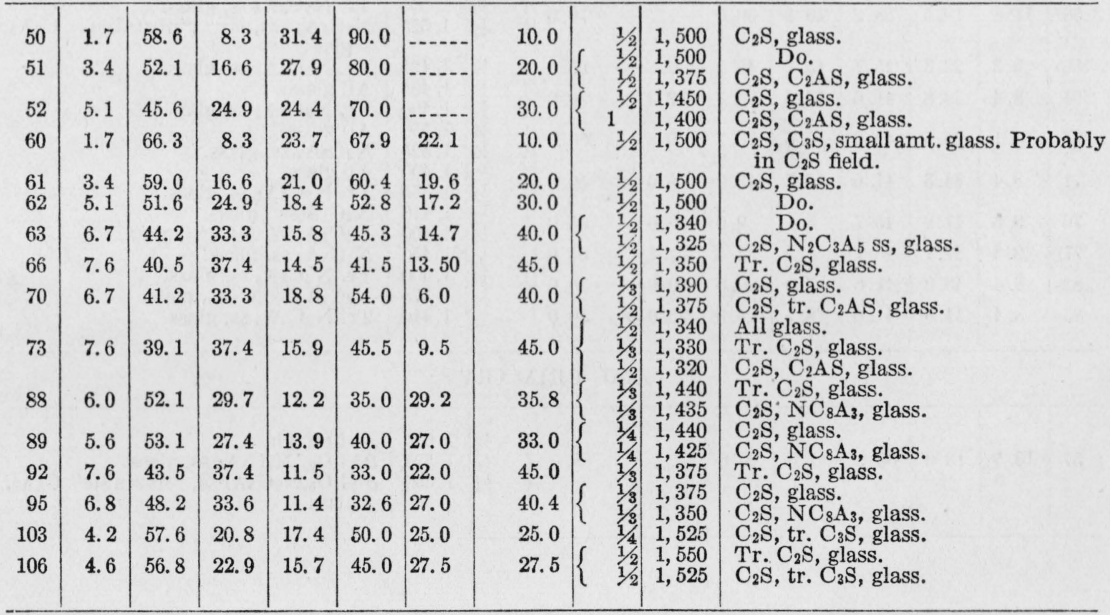

\section{$\mathrm{NC}_{8} \mathrm{~A}_{3}$ PRIMARY}

\begin{tabular}{|c|c|c|c|c|c|c|c|c|c|c|}
\hline 74 & 9.3 & 45.0 & 45.7 & $\ldots$ & - & 45.0 & 55.0 & $1 / 2$ & 1,470 & $\mathrm{NC}_{8} \mathrm{~A}_{3}$, glass. \\
\hline 75 & 9.3 & 43.4 & 45.7 & 1. 6 & 4.5 & 40.5 & 55.0 & $\left\{\begin{array}{l}1 / 3 \\
1 / 3\end{array} \mid\right.$ & $\begin{array}{l}1,450 \\
1,445\end{array}$ & $\begin{array}{l}\text { All glass. } \\
\mathrm{NC}_{8} \mathrm{~A}_{3} \text {, small amt. } \mathrm{N}_{2} \mathrm{C}_{3} \mathrm{~A}_{5} \text {, glass. } \\
\text { Near boundary. }\end{array}$ \\
\hline 80 & 8.4 & 46.5 & 41.6 & 3.5 & 10.0 & 40.0 & 50.0 & $\left\{\begin{array}{l}1 / 3 \\
1 / 3\end{array}\right.$ & $\begin{array}{l}1,460 \\
1,455\end{array}$ & $\begin{array}{l}\text { All glass. } \\
\mathrm{NC}_{8} \mathrm{~A}_{3}, \text { glass. }\end{array}$ \\
\hline 81 & 8.4 & 44.8 & 41.6 & 5.2 & 15.0 & 35.0 & 50.0 & $\begin{array}{l}1 / 3 \\
1 / 3\end{array}$ & $\begin{array}{l}1,425 \\
1,420\end{array}$ & $\begin{array}{l}\text { All glass. } \\
\mathrm{NC}_{8} \mathrm{~A}_{3}, \text { glass. }\end{array}$ \\
\hline 85 & 7.4 & 49.0 & 36.6 & 7.0 & 20.0 & 36.0 & 44.0 & $1 / 2$ & 1,460 & Tr. $\mathrm{NC}_{8} \mathrm{~A}_{3}$, glass. \\
\hline 87 & 6.5 & 51.0 & 32.0 & 10.5 & 30.0 & 31.5 & 38.5 & $\left\{\begin{array}{l}1 / 4 \\
1 / 3\end{array}\right.$ & $\begin{array}{l}1,450 \\
1,440\end{array}$ & $\begin{array}{l}\text { All glass. } \\
\mathrm{NC}_{8} \mathrm{~A}_{\mathbf{8}}, \text { glass. }\end{array}$ \\
\hline
\end{tabular}


TABLE 2.-Quenching data for compositions in the $2 \mathrm{CaO} \cdot \mathrm{SiO}_{2}-\mathrm{CaO}-\left(\mathrm{Na}_{2} \mathrm{O}+3 \mathrm{Al}_{2} \mathrm{O}_{3}\right)$ plane-Continued

\begin{tabular}{|c|c|c|c|c|c|c|c|c|c|c|}
\hline \multirow{3}{*}{ No. } & \multicolumn{7}{|c|}{ Composition } & \multirow{3}{*}{ Time } & \multirow{3}{*}{$\begin{array}{l}\text { Tem- } \\
\text { pera- } \\
\text { ture }\end{array}$} & \multirow{3}{*}{ Phases present } \\
\hline & \multicolumn{4}{|c|}{ Oxides } & \multicolumn{3}{|c|}{ Components } & & & \\
\hline & $\mathrm{Na}_{2} \mathrm{O}$ & $\mathrm{CaO}$ & $\mathrm{Al}_{2} \mathrm{O}_{3}$ & $\mathrm{SiO}_{2}$ & $\mathrm{C}_{2} \mathrm{~S}$ & $\mathrm{CaO}$ & $\begin{array}{l}\left(\mathrm{Na}_{2} \mathrm{O}+\right. \\
\left.3 \mathrm{Al}_{2} \mathrm{O}_{3}\right)\end{array}$ & & & \\
\hline
\end{tabular}

\section{$\mathrm{C}_{5} \mathrm{~A}_{3}$ PRIMARY}

\begin{tabular}{|c|c|c|c|c|c|c|c|c|c|c|}
\hline & $\%$ & $\%$ & $\%$ & $\%$ & $\%$ & $\%$ & $\%$ & & ${ }^{\circ} \mathrm{C}$ & All glogs \\
\hline 91 & 7.6 & 45.4 & 37.4 & 9.6 & 27. 5 & 27.5 & 45.0 & $\begin{array}{l}1 / 2 \\
1 / 3\end{array}$ & $\begin{array}{l}1,360 \\
1,355\end{array}$ & Tr. $\mathrm{C}_{5} \mathrm{~A}_{3}$ glass. \\
\hline 93 & 7.6 & 44.4 & 37.4 & 10.6 & 30.3 & 24.7 & 45.0 & $\begin{array}{l}1 / 3 \\
1 / 2 \\
1 / 2\end{array}$ & $\begin{array}{l}1,360 \\
1,345 \\
1,335\end{array}$ & $\begin{array}{l}\mathrm{C}_{5} \mathrm{~A}_{3}, \text { tr. } \mathrm{C}_{2} \mathrm{~S} \text {, glass. } \\
\mathrm{C}_{5} \mathrm{~A}_{3}, \mathrm{C}_{2} \mathrm{~S}, \mathrm{~N}_{2} \mathrm{C}_{3} \mathrm{~A}_{5} \mathrm{Ss} \text {, glass. } \\
\text { All crystalline. }\end{array}$ \\
\hline 94 & 7.8 & 44.4 & 38.4 & 9.4 & 26.9 & 26.9 & 46. 2 & $1 / 3$ & $\begin{array}{l}1,370 \\
1,360\end{array}$ & $\begin{array}{l}\text { All glass. } \\
\mathrm{C}_{5} \mathrm{~A}_{3}, \text { glass. }\end{array}$ \\
\hline
\end{tabular}

$\mathrm{C}_{2} \mathrm{AS}$ PRIMARY

\begin{tabular}{|c|c|c|c|c|c|c|c|c|c|c|}
\hline 53 & 6.7 & 39.1 & 33.3 & 20.9 & 60.0 & & 40.0 & $\begin{array}{l}1 / 2 \\
1 / 2\end{array}$ & $\begin{array}{l}1,430 \\
1,420\end{array}$ & $\begin{array}{l}\text { All glass. } \\
\mathrm{C}_{2} \mathrm{AS} \text {, glass. }\end{array}$ \\
\hline 54 & 8.4 & 32.6 & 41. 6 & 17. 4 & 50.0 & & 50.0 & $1 / 2$ & $\begin{array}{l}1,420 \\
1,415\end{array}$ & $\begin{array}{l}\text { All glass. } \\
\mathrm{C}_{2} \mathrm{AS} \text {, glass. }\end{array}$ \\
\hline 59 & 7.6 & 35.8 & 37.4 & 19.2 & 55.0 & & 45.0 & $\begin{array}{l}1 / 2 \\
1 / 3\end{array}$ & $\begin{array}{l}1,435 \\
1,425\end{array}$ & $\begin{array}{l}\text { All glass. } \\
\mathrm{C}_{2} \mathrm{AS} \text {, glass. }\end{array}$ \\
\hline 72 & 7.6 & 37.7 & 37.4 & 17.3 & 49.5 & 5.5 & 45.0 & $\begin{array}{l}1 / 2 \\
1 / 2\end{array}$ & $\begin{array}{l}1,395 \\
1,385\end{array}$ & $\begin{array}{l}\text { All glass. } \\
\mathrm{C}_{2} \mathrm{AS} \text {, glass. }\end{array}$ \\
\hline
\end{tabular}

\section{$\mathrm{N}_{2} \mathrm{C}_{3} \mathrm{~A}_{5}$ SOLID SOLUTION PRIMARY}

\begin{tabular}{|c|c|c|c|c|c|c|c|c|c|c|c|}
\hline 55 & 10.1 & 26.1 & 49.9 & 13.9 & 40.0 & & 60.0 & $1 / 2$ & 1,500 & Tr. $\mathrm{N}_{2} \mathrm{C}_{3} \mathrm{~A}_{5}$ ss, glass. & \\
\hline 56 & 11.8 & 19.5 & 58.2 & 10.5 & 30.0 & $-\cdots$ & 70.0 & $\begin{array}{l}1 / 6 \\
1 / 3\end{array}$ & $\begin{array}{l}1,565 \\
1,525\end{array}$ & $\begin{array}{l}\mathrm{Tr} . \mathrm{N}_{2} \mathrm{C}_{3} \mathrm{~A}_{5} \text { Ss, glass. } \\
\mathrm{N}_{2} \mathrm{C}_{3} \mathrm{~A}_{5} \text { ss, tr. "unstable" }\end{array}$ & $\mathrm{C}_{3} \mathrm{~A}_{5}$ \\
\hline 58 & 9.3 & 29.3 & 45.7 & 15. 7 & 45.0 & $-\ldots$ & 55.0 & $1 / 3$ & 1,425 & Tr. $\mathrm{N}_{2} \mathrm{C}_{3} \mathrm{~A}_{5} \mathrm{ss}$, glass. & \\
\hline 64 & 8.4 & 36.8 & 41.6 & 13.2 & 33.7 & 12.3 & 50.0 & $\begin{array}{l}1 / 2 \\
1 / 2\end{array}$ & $\begin{array}{l}1,405 \\
1,400\end{array}$ & $\begin{array}{l}\text { All glass. } \\
\text { Tr. } \mathrm{N}_{2} \mathrm{C}_{3} \mathrm{~A}_{5} \mathrm{ss} \text {, glass. }\end{array}$ & \\
\hline 65 & 10.1 & 29.5 & 49.9 & 10.5 & 30.2 & 9.8 & 60.0 & $\begin{array}{l}1 / 6 \\
1 / 4\end{array}$ & $\begin{array}{l}1,550 \\
1,525\end{array}$ & $\begin{array}{l}\text { All glass. } \\
\mathrm{N}_{2} \mathrm{C}_{3} \mathrm{~A}_{5} \text { ss, glass. }\end{array}$ & \\
\hline 71 & 8.4 & 34.3 & 41. 6 & 15. 7 & 45.0 & 5.0 & 50.0 & $\begin{array}{l}1 / 2 \\
1 / 2\end{array}$ & $\begin{array}{l}1,400 \\
1,375\end{array}$ & $\begin{array}{l}\text { All glass. } \\
\text { Tr. } \mathrm{N}_{2} \mathrm{C}_{3} \mathrm{~A}_{5} \text { SS, glass. }\end{array}$ & \\
\hline 76 & 9.3 & 41.9 & 45.7 & 3.1 & 9.0 & 36.0 & 55.0 & $\begin{array}{l}1 / 2 \\
1 / 3\end{array}$ & $\begin{array}{l}1,410 \\
1,400\end{array}$ & $\begin{array}{l}\mathrm{N}_{2} \mathrm{C}_{3} \mathrm{~A}_{5} \mathrm{SS}, \text { glass. } \\
\mathrm{N}_{2} \mathrm{C}_{3} \mathrm{~A}_{5} \mathrm{ss}, \mathrm{NC}_{8} \mathrm{~A}_{3} \text {, glass. }\end{array}$ & \\
\hline 77 & 9.3 & 37.1 & 45.7 & 7. 9 & 22.5 & 22.5 & 55.0 & $1 / 3$ & 1,450 & $\mathrm{~N}_{2} \mathrm{C}_{3} \mathrm{~A}_{5}$ ss, glass. & \\
\hline 82 & 8.4 & 43.0 & 41. 6 & 7. 0 & 20.0 & 30.0 & 50.0 & $\begin{array}{l}1 / 3 \\
1 / 3\end{array}$ & $\begin{array}{l}1,400 \\
1,375\end{array}$ & $\begin{array}{l}\mathrm{Tr} . \mathrm{N}_{2} \mathrm{C}_{3} \mathrm{~A}_{5} \text { ss, glass. } \\
\mathrm{N}_{2} \mathrm{C}_{3} \mathrm{~A}_{5} \mathrm{ss}, \mathrm{C}_{5} \mathrm{~A}_{3} \text {, glass. }\end{array}$ & \\
\hline 83 & 8.4 & 41.3 & 41. 6 & 8. 7 & 25.0 & 25.0 & 50.0 & $1 / 3$ & 1,410 & Tr. $\mathrm{N}_{2} \mathrm{C}_{3} \mathrm{~A}_{5}$ ss, glass. & \\
\hline
\end{tabular}

$\mathrm{Al}_{2} \mathrm{O}_{3}$ PRIMARY

\begin{tabular}{|c|c|c|c|c|c|c|c|c|c|c|}
\hline 57 & 13.5 & 13.0 & 66.5 & 7.0 & 20.0 & -...... & 80.0 & $\begin{array}{l}1 / 6 \\
1 / 6 \\
1 / 4\end{array}$ & $\begin{array}{l}1,600 \\
1,575 \\
1,550\end{array}$ & $\begin{array}{l}\boldsymbol{\beta A l _ { 2 } \mathrm { O } _ { 3 }} \text {, glass. } \\
\boldsymbol{\beta A l}_{2} \mathrm{O}_{3}, \mathrm{~N}_{2} \mathrm{C}_{3} \mathrm{~A}_{5} \mathrm{ss} \text {, glass. } \\
\boldsymbol{\beta A l}_{2} \mathrm{O}_{3}, \mathrm{~N}_{2} \mathrm{C}_{3} \mathrm{~A}_{5} \mathrm{ss} \text {, "unstable" } \mathrm{C}_{3} \mathrm{~A}_{5}, \\
\quad \text { glass. }\end{array}$ \\
\hline
\end{tabular}


TABLE 3.-Quenching data for compositions in the system $2 \mathrm{CaO} . \mathrm{SiO}_{2}-\mathrm{CaO}-\mathrm{Na}_{2} \mathrm{O} . \mathrm{Al}_{2} \mathrm{O}_{3}$

\begin{tabular}{|c|c|c|c|c|c|c|c|c|c|c|}
\hline \multirow{3}{*}{ No. } & \multicolumn{7}{|c|}{ Composition } & \multirow{3}{*}{ Time } & \multirow{3}{*}{$\begin{array}{l}\text { Tem- } \\
\text { pera- } \\
\text { ture }\end{array}$} & \multirow{3}{*}{ Phases present } \\
\hline & \multicolumn{4}{|c|}{ Oxides } & \multicolumn{3}{|c|}{ Components } & & & \\
\hline & $\mathrm{Na}_{2} \mathrm{O}$ & $\mathrm{CaO}$ & $\mathrm{Al}_{2} \mathrm{O}_{3}$ & $\mathrm{SiO}_{2}$ & $\mathrm{C}_{2} \mathrm{~S}$ & $\mathrm{CaO}$ & NA & & & \\
\hline
\end{tabular}

CaO PRIMARY

\begin{tabular}{|c|c|c|c|c|c|c|c|c|c|c|}
\hline & $\%$ & $\%$ & $\%$ & $\%$ & $\%$ & $\%$ & $\%$ & $h r$ & ${ }^{\circ} \mathrm{C}$ & \\
\hline 37 & 3.8 & 68.0 & 6. 2 & 22.0 & 63.0 & 27.0 & 10.0 & & 1,550 & $\mathrm{CaO}, \mathrm{C}_{3} \mathrm{~S}$, glass. \\
\hline 39 & 7. 6 & 60.5 & 12. 4 & 19.5 & 56.0 & 24.0 & 20.0 & $1 / 2$ & $\begin{array}{l}1,550 \\
1,500\end{array}$ & $\begin{array}{l}\mathrm{CaO} \text {, tr. } \mathrm{C}_{2} \mathrm{~S} \text {, glass. } \\
\mathrm{CaO} \text {, tr. } \mathrm{NA} \text {, glass. }\end{array}$ \\
\hline 41 & 11. 3 & 52.9 & 18. 7 & 17.1 & 49.0 & 21.0 & 30.0 & & $\begin{array}{l}1,550 \\
1,500\end{array}$ & $\begin{array}{l}\text { Small amt. CaO, quench growths. } \\
\text { glass. } \\
\mathrm{CaO} \text {, NA, quench growths, glass. }\end{array}$ \\
\hline
\end{tabular}

$\mathrm{C}_{3} \mathrm{~S}$ PRIMARY

\begin{tabular}{|c|c|c|c|c|c|c|c|c|c|c|}
\hline 18 & 7.6 & 59.0 & 12. 4 & 21. 0 & 60.4 & 19.6 & 20.0 & $\begin{array}{l}1 / 3 \\
1 / 3\end{array}$ & $\begin{array}{l}1,600 \\
1,550\end{array}$ & $\begin{array}{l}\mathrm{C}_{3} \mathrm{~S} \text {, quench growths, glass. } \\
\mathrm{C}_{3} \mathrm{~S} \text {, small amt. } \mathrm{C}_{2} \mathrm{~S} \text {, glass. }\end{array}$ \\
\hline 20 & 5. 7 & 62.6 & 9.3 & 22.4 & 64.1 & 20.9 & 15. 0 & $\begin{array}{l}1 / 3 \\
1\end{array}$ & $\begin{array}{l}1,550 \\
1,500\end{array}$ & $\begin{array}{l}\mathrm{C}_{3} \mathrm{~S} \text {, small amt. } \mathrm{C}_{2} \mathrm{~S} \text {, tr. } \mathrm{CaO} \text {, glass. } \\
\mathrm{C}_{3} \mathrm{~S} \text {, small amt. } \mathrm{C}_{2} \mathrm{~S} \text { and } \mathrm{CaO} \text {, glass. }\end{array}$ \\
\hline
\end{tabular}

$\mathrm{C}_{2} \mathrm{~S}$ PRIMARY

\begin{tabular}{|c|c|c|c|c|c|c|c|c|c|c|}
\hline $\begin{array}{l}12 \\
13 \\
14\end{array}$ & $\begin{array}{r}3.8 \\
7.6 \\
11.3\end{array}$ & $\begin{array}{l}58.6 \\
52.1 \\
45.6\end{array}$ & $\begin{array}{r}6.2 \\
12.4 \\
18.7\end{array}$ & $\begin{array}{l}31.4 \\
27.9 \\
24.4\end{array}$ & $\begin{array}{l}90.0 \\
80.0 \\
70.0\end{array}$ & 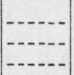 & $\begin{array}{l}10.0 \\
20.0 \\
30.0\end{array}$ & $\begin{array}{l}1 / 2 \\
1 / 2 \\
1 / 3\end{array}$ & $\begin{array}{l}1,500 \\
1,550 \\
1,550\end{array}$ & $\begin{array}{l}\mathrm{C}_{2} \mathrm{~S} \text {, small amt. glass. } \\
\mathrm{C}_{2} \mathrm{~S} \text {, glass. } \\
\text { Tr. } \mathrm{C}_{2} \mathrm{~S} \text {, quench growths, glass. }\end{array}$ \\
\hline 24 & 3.8 & 61.8 & 6.2 & 28.2 & 81.0 & 9.0 & 10.0 & $\begin{array}{l}1 / 4 \\
1,3\end{array}$ & & \\
\hline 25 & 7. 6 & 54.9 & 12.4 & 25.1 & 72.0 & 8.0 & 20.0 & 1 & 1,500 & $\mathrm{C}_{2} \mathrm{~S}$, glass. \\
\hline
\end{tabular}

$\mathrm{C}_{2} \mathrm{~S}$ PRIMARY

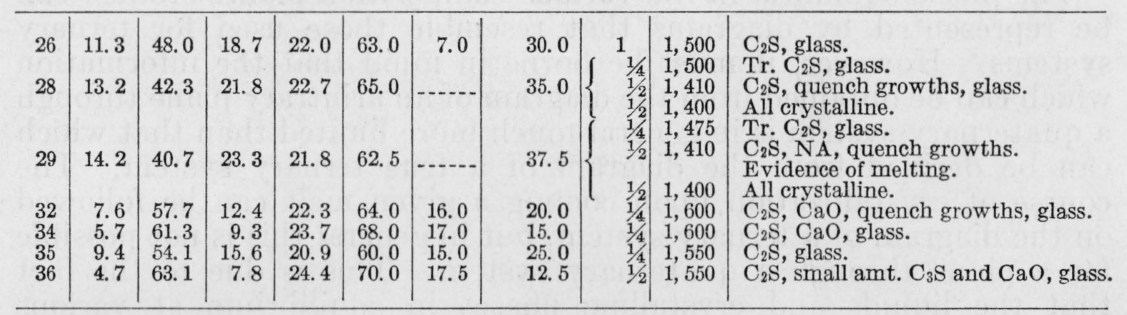

\section{NA PRIMARY}

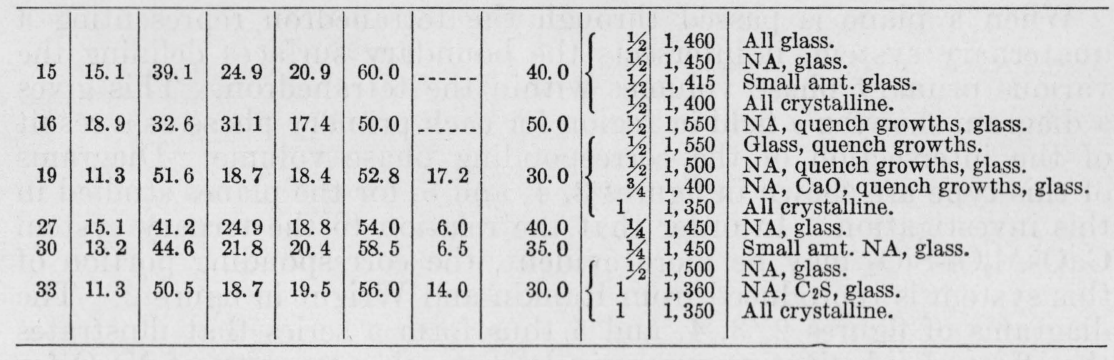


TABLE 4.-Quenching data for additional compositions located between the $2 \mathrm{CaO} . \mathrm{SiO}_{2}-\mathrm{CaO}-\left(\mathrm{Na}_{2} \mathrm{O}+6 \mathrm{Al}_{2} \mathrm{O}_{3}\right)$ and $2 \mathrm{CaO} . \mathrm{SiO}_{2}-\mathrm{CaO}-\left(\mathrm{Na}_{2} \mathrm{O}+3 \mathrm{Al}_{2} \mathrm{O}_{3}\right)$ planes

\begin{tabular}{|c|c|c|c|c|c|c|c|c|c|c|c|}
\hline \multirow{3}{*}{ No. } & \multicolumn{8}{|c|}{ Composition } & \multirow{3}{*}{ Time } & \multirow{3}{*}{$\begin{array}{l}\text { Tem- } \\
\text { pera- } \\
\text { ture }\end{array}$} & \multirow{3}{*}{ Phases present } \\
\hline & \multicolumn{4}{|c|}{ Oxides } & \multicolumn{4}{|c|}{ Components } & & & \\
\hline & $\mathrm{Na}_{2} \mathrm{O}$ & $\mathrm{CaO}$ & $\mathrm{Al}_{2} \mathrm{O}_{3}$ & $\mathrm{SiO}_{2}$ & $\mathrm{C}_{2} \mathrm{~S}$ & $\mathrm{CaO}$ & $\begin{array}{l}\left(\mathrm{Na}_{2} \mathrm{O}+\right. \\
\left.6 \mathrm{Al}_{2} \mathrm{O}_{3}\right)\end{array}$ & $\begin{array}{l}\left(\mathrm{Na}_{2} \mathrm{O}+\right. \\
\left.3 \mathrm{Al}_{2} \mathrm{O}_{3}\right)\end{array}$ & & & \\
\hline & $\%$ & $\%$ & $\%$ & $\%$ & $\%$ & $\%$ & $\%$ & $\%$ & $h r$ & ${ }^{\circ} \mathrm{C}$ & \\
\hline 200 & 4.4 & 53.7 & 29.7 & 12.2 & 35.0 & 30.9 & 17.1 & 17.0 & $\left\{\begin{array}{l}1 / 3 \\
1 / 3\end{array}\right.$ & $\begin{array}{l}1,435 \\
1,430\end{array}$ & $\begin{array}{l}\mathrm{C}_{2} \mathrm{~S} \text {, glass. } \\
\mathrm{C}_{2} \mathrm{~S} \text {, small amt. } \mathrm{NC}_{8} \mathrm{~A}_{3} \text {, } \\
\text { glass. }\end{array}$ \\
\hline 201 & 3.8 & 53.7 & 30.3 & 12.2 & 35.0 & 30.9 & 25.6 & 8.5 & $\begin{array}{l}1 / 2 \\
1 / 3 \\
1 / 3 \\
11 / 4 \\
1 / 4\end{array}$ & $\begin{array}{l}1,425 \\
1,420 \\
1,415 \\
1,450 \\
1,440\end{array}$ & $\begin{array}{l}\mathrm{C}_{2} \mathrm{~S} \text {, glass. } \\
\mathrm{C}_{2} \mathrm{~S} \text {, tr. } \mathrm{NC}_{8} \mathrm{~A}_{3} \text {, glass. } \\
\mathrm{C}_{2} \mathrm{~S} \text {, NC } \mathrm{NA}_{3} \text {, glass. } \\
\mathrm{C}_{2} \mathrm{~S} \text {, CaO, tr. } \mathrm{C}_{3} \mathrm{~S} \text {, glass. } \\
\mathrm{C}_{2} \mathrm{~S} \text {, small amt. N } \mathrm{C}_{8} \mathrm{~A}_{3} \text {, tr. } \\
\mathrm{C}_{3} \mathrm{~S} \text {, glass. } \mathrm{C}_{3} \mathrm{~A} \text { not ob- }\end{array}$ \\
\hline 202 & 4.2 & 00.0 & 20.0 & 12.2 & 30.0 & 32.0 & 10.0 & 10.2 & $1 / 4$ & 1,435 & $\begin{array}{l}\text { served. } \\
\mathrm{C}_{2} \mathrm{~S} \text {, } \mathrm{NC}_{8} \mathrm{~A}_{3} \text {, glass. } \mathrm{C}_{3} \mathrm{~A} \\
\text { not observed. }\end{array}$ \\
\hline 203 & 3.6 & 55.3 & 28.9 & 12.2 & 35.0 & 32.5 & 24.4 & 8.1 & $\begin{array}{l}1 / 4 \\
1 / 4 \\
11 / 4 \\
1 / 4\end{array}$ & $\begin{array}{l}1,445 \\
1,440 \\
1,435 \\
1,445\end{array}$ & $\begin{array}{l}\mathrm{C}_{2} \mathrm{~S} \text {, glass. } \\
\mathrm{C}_{2} \mathrm{~S}, \mathrm{C}_{3} \mathrm{~A}, \text { glass. } \\
\mathrm{C}_{2} \mathrm{~S}, \mathrm{C}_{3} \mathrm{~A}, \mathrm{NC}_{8} \mathrm{~A}_{3} \text {, glass. } \\
\text { All glass. }\end{array}$ \\
\hline 204 & 3.9 & 54.4 & 31.1 & 10.5 & 30.0 & 35.0 & 26.3 & 8.7 & $\begin{array}{l}1 / 4 \\
1 / 4\end{array}$ & $\begin{array}{l}1,440 \\
1,435\end{array}$ & $\begin{array}{l}\mathrm{NC}_{8} \mathrm{~A}_{3}, \text { possibly } \mathrm{C}_{3} \mathrm{~A} \text {, glass. } \\
\mathrm{NC}_{8} \mathrm{~A}_{3}, \mathrm{C}_{2} \mathrm{~S} \text {, possibly } \mathrm{C}_{3} \mathrm{~A} \text {, } \\
\text { glass. }\end{array}$ \\
\hline 205 & 3.8 & 55.3 & 30.6 & 10.3 & 29.5 & 36.0 & 25.9 & 8.6 & $\left\{\begin{array}{l}1 / 4 \\
1 / 3 \\
1 / 4\end{array}\right.$ & $\begin{array}{l}1,450 \\
1,445 \\
1,435\end{array}$ & $\begin{array}{l}\mathrm{Tr} . \mathrm{C}_{3} \mathrm{~S}, \text { tr. } \mathrm{CaO} \text {, glass. } \\
\mathrm{C}_{3} \mathrm{~S} \text {, tr. } \mathrm{CaO} \text {, glass. } \\
\mathrm{C}_{2} \mathrm{~S}, \mathrm{NC}_{8} \mathrm{~A}_{3} \text {, probably } \mathrm{C}_{3} \mathrm{~A} \text {, } \\
\text { glass. }\end{array}$ \\
\hline 206 & 3.5 & 55.2 & 31.0 & 10. 3 & 29.5 & 36.0 & 30.2 & 4.3 & $\begin{array}{l}1 / 4 \\
1 / 2 \\
1 / 2\end{array}$ & $\begin{array}{l}1,450 \\
1,440 \\
1,430\end{array}$ & $\begin{array}{l}\text { Small amt. } \mathrm{C}_{3} \mathrm{~A}, \text { tr. } \mathrm{C}_{3} \mathrm{~S}, \\
\text { tr. } \mathrm{CaO}_{2} \mathrm{O} \text {, glass. } \\
\mathrm{C}_{2} \mathrm{~S}, \mathrm{C}_{3} \mathrm{~A}, \mathrm{C}_{3} \mathrm{~S}, \mathrm{NC}_{8} \mathrm{~A}_{3} \text {, } \\
\text { glass. } \\
\mathrm{C}_{2} \mathrm{~S}, \mathrm{C}_{3} \mathrm{~A}, \mathrm{NC}_{8} \mathrm{~A}_{3} \text {, glass. }\end{array}$ \\
\hline
\end{tabular}

The phase equilibria in the various composition planes studied can be represented by diagrams that resemble those used for ternary systems. However, it must be borne in mind that the information which can be obtained from the diagram of an arbitrary plane through a quaternary system is in general much more limited than that which can be derived from the diagram of a true ternary system. The course of crystallization upon cooling a given melt can be followed on the diagram of a ternary system, but in general this is not possible for a plane through a quaternary system. This is due to the fact that the liquids and crystalline phases in equilibrium at various temperatures usually have compositions outside of the original plane.

When a plane is passed through the tetrahedron representing a quaternary system, it intersects the boundary surfaces defining the various primary phase volumes within the tetrahedron. This gives a diagram showing a field or region for each primary phase as a result of the intersection of the corresponding phase volume. Diagrams of this type are shown in figures 3,4 , and 5 , for the planes studied in this investigation. In order that the relation to the ternary system $\mathrm{CaO}-\mathrm{Al}_{2} \mathrm{O}_{3}-\mathrm{SiO}_{2}$ may be more evident, the corresponding portion of this system is reproduced from Rankin and Wright in figure 2. The diagrams of figures $2,3,4$, and 5 thus form a series that illustrates the effect of substituting successively increasing amounts of $\mathrm{Na}_{2} \mathrm{O}$ for $\mathrm{Al}_{2} \mathrm{O}_{3}$ in the system $\mathrm{CaO}-\mathrm{Al}_{2} \mathrm{O}_{3}-\mathrm{SiO}_{2}$. As mentioned previously, the ratio of $\mathrm{Na}_{2} \mathrm{O}$ to $\mathrm{Al}_{2} \mathrm{O}_{3}$ remains constant in any particular plane.

As the calcium silicates $2 \mathrm{CaO} . \mathrm{SiO}_{2}$ and $3 \mathrm{CaO} . \mathrm{SiO}_{2}$ are major crystalline phases of portland cement clinker, the interpretation of 
the system $\mathrm{Na}_{2} \mathrm{O}-\mathrm{CaO}-\mathrm{Al}_{2} \mathrm{O}_{3}-\mathrm{SiO}_{2}$ in relation to cement is simplified by keeping $2 \mathrm{CaO} \cdot \mathrm{SiO}_{2}$ and $\mathrm{CaO}$ at two of the apexes of each composition plane.

\section{THE PLANE $2 \mathrm{CaO} . \mathrm{SiO}_{2}-\mathrm{CaO}-\left(\mathrm{Na}_{2} \mathrm{O}+6 \mathrm{Al}_{2} \mathrm{O}_{3}\right)$}

The diagram of this plane is shown in figure 3 , and is based on the data of table 1 . There is considerable resemblance to the corresponding part of the $\mathrm{CaO}-\mathrm{Al}_{2} \mathrm{O}_{3}-\mathrm{SiO}_{2}$ system (fig. 2), with the differ-

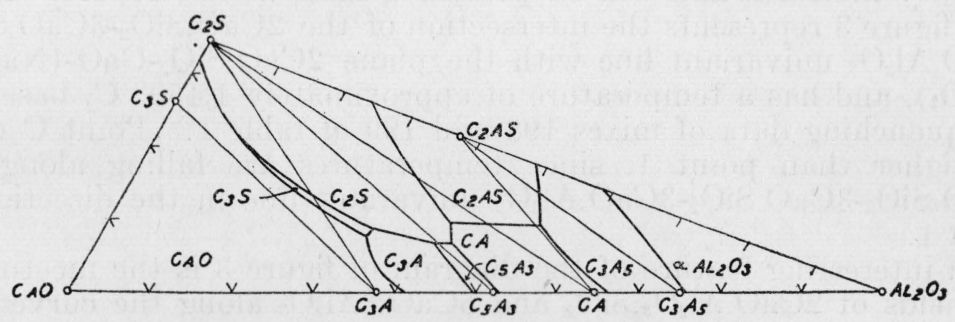

Figure 2.-Part of system $\mathrm{CaO}-\mathrm{Al}_{2} \mathrm{O}_{3}-\mathrm{SiO}_{2}$.

ence that two additional crystalline phases have made their appearance. These are the $\mathrm{Na}_{2} \mathrm{O}$-bearing compounds, $\mathrm{Na}_{2} \mathrm{O} .8 \mathrm{CaO} .3 \mathrm{Al}_{2} \mathrm{O}_{3}$ and $2 \mathrm{Na}_{2} \mathrm{O} .3 \mathrm{CaO} .5 \mathrm{Al}_{2} \mathrm{O}_{3}$. A total of 11 primary phase regions are indicated. The others are those of $\mathrm{CaO}, 3 \mathrm{CaO} . \mathrm{SiO}_{2}, 2 \mathrm{CaO} . \mathrm{SiO}_{2}$, $3 \mathrm{CaO} . \mathrm{Al}_{2} \mathrm{O}_{3}, 5 \mathrm{CaO} .3 \mathrm{Al}_{2} \mathrm{O}_{3}, 2 \mathrm{CaO} . \mathrm{Al}_{2} \mathrm{O}_{3} \cdot \mathrm{SiO}_{2}, \mathrm{CaO} \cdot \mathrm{Al}_{2} \mathrm{O}_{3}, 3 \mathrm{CaO} .5 \mathrm{Al}_{2} \mathrm{O}_{3}$, and $\alpha-\mathrm{Al}_{2} \mathrm{O}_{3}$.

The area in which $\mathrm{Na}_{2} \mathrm{O} .8 \mathrm{CaO} \cdot 3 \mathrm{Al}_{2} \mathrm{O}_{3}$ is the primary phase is seen to be very narrow. In the neighborhood of points $\mathrm{A}$ and $\mathrm{B}$ (fig. 3 )

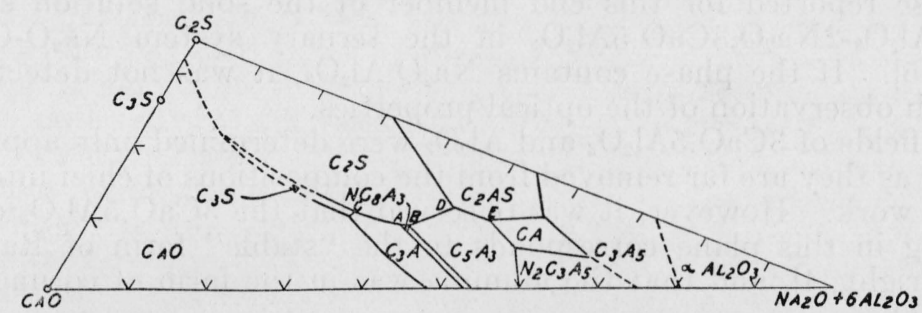

Figure 3.-Composition plane $2 \mathrm{CaO} \cdot \mathrm{SiO}_{2}-\mathrm{CaO}-\left(\mathrm{Na}_{2} \mathrm{O}+6 \mathrm{Al}_{2} \mathrm{O}_{3}\right)$.

the fields of four crystalline phases meet nearly in a single point. The composition where this actually occurs is the invariant point for $2 \mathrm{CaO} . \mathrm{SiO}_{2}, 3 \mathrm{CaO} . \mathrm{Al}_{2} \mathrm{O}_{3}, 5 \mathrm{CaO} .3 \mathrm{Al}_{2} \mathrm{O}_{3}, \mathrm{Na}_{2} \mathrm{O} .8 \mathrm{CaO} .3 \mathrm{Al}_{2} \mathrm{O}_{3}$, and liquid. It is located slightly outside the plane of the diagram in the direction of lower $\mathrm{Na}_{2} \mathrm{O} / \mathrm{Al}_{2} \mathrm{O}_{3}$ ratios. The composition of this point, designated invariant point 4 , is estimated to be 3.5 percent $\mathrm{Na}_{2} \mathrm{O}$, 50.2 percent $\mathrm{CaO}, 37.6$ percent $\mathrm{Al}_{2} \mathrm{O}_{3}$, and 8.7 percent $\mathrm{SiO}_{2}$. The temperature is $1,365^{\circ} \pm 10^{\circ} \mathrm{C}$. It is not a eutectic. Mix 154 of table 1 is near its composition. The temperature of $1,365^{\circ} \mathrm{C}$ for point 4 is worthy of note, as it is higher, rather than lower, than the corresponding ternary invariant point in the system $\mathrm{CaO}-\mathrm{Al}_{2} \mathrm{O}_{3}-\mathrm{SiO}_{2}$. The $2 \mathrm{CaO} . \mathrm{SiO}_{2}-3 \mathrm{CaO} . \mathrm{Al}_{2} \mathrm{O}_{3}-5 \mathrm{CaO} .3 \mathrm{Al}_{2} \mathrm{O}_{3}$ eutectic in the $\mathrm{CaO}-\mathrm{Al}_{2} \mathrm{O}_{3}$ $\mathrm{SiO}_{2}$ system is at $1,335^{\circ} \mathrm{C}$ [4]. The explanation for this appears to be the fact that the $5 \mathrm{CaO}_{3} . \mathrm{Al}_{2} \mathrm{O}_{3}$ in the quaternary system is not 
pure, but contains $\mathrm{Na}_{2} \mathrm{O}$ in solid solution. An analogous situation was found by Brownmiller and Bogue in the ternary system $\mathrm{Na}_{2} \mathrm{O}$ $\mathrm{CaO}-\mathrm{Al}_{2} \mathrm{O}_{3}[5]$.

The important invariant point, not a eutectic, where $2 \mathrm{CaO} \cdot \mathrm{SiO}_{2}$, $3 \mathrm{CaO} . \mathrm{SiO}_{2}, 3 \mathrm{CaO} . \mathrm{Al}_{2} \mathrm{O}_{3}$, and $\mathrm{Na}_{2} \mathrm{O} .8 \mathrm{CaO} .3 \mathrm{Al}_{2} \mathrm{O}_{3}$ are in equilibrium with liquid, designated invariant point 1 , also lies outside of this plane, but in the direction of higher $\mathrm{Na}_{2} \mathrm{O} / \mathrm{Al}_{2} \mathrm{O}_{3}$ ratios. Its composition and temperature were established by determinations on supplementary mixtures and will be presented later in this report. Point $\mathrm{C}$ of figure 3 represents the intersection of the $2 \mathrm{CaO} . \mathrm{SiO}_{2}-3 \mathrm{CaO} . \mathrm{SiO}_{2}-$ $3 \mathrm{CaO} . \mathrm{Al}_{2} \mathrm{O}_{3}$ univariant line with the plane $2 \mathrm{CaO} \cdot \mathrm{SiO}_{2}-\mathrm{CaO}-\left(\mathrm{Na}_{2} \mathrm{O}+\right.$ $6 \mathrm{Al}_{2} \mathrm{O}_{3}$ ), and has a temperature of approximately $1,450^{\circ} \mathrm{C}$, based on the quenching data of mixes 198 and 199 of table 1 . Point $\mathrm{C}$ must be higher than point 1 , since temperatures are falling along the $2 \mathrm{CaO} . \mathrm{SiO}_{2}-3 \mathrm{CaO} . \mathrm{SiO}_{2}-3 \mathrm{CaO} \cdot \mathrm{Al}_{2} \mathrm{O}_{3}$ univariant line in the direction of point 1.

An interesting feature of the diagram of figure 3 is the meeting of the fields of $2 \mathrm{CaO} \cdot \mathrm{Al}_{2} \mathrm{O}_{3} \cdot \mathrm{SiO}_{2}$ and $5 \mathrm{CaO} \cdot 3 \mathrm{Al}_{2} \mathrm{O}_{3}$ along the curve DE. This is unexpected as these two compounds are incompatible in the system $\mathrm{CaO}-\mathrm{Al}_{2} \mathrm{O}_{3}-\mathrm{SiO}_{2}$, as is evident in figure 2 . One possible explanation for this behavior is the taking up in solid solution of about 1 percent of $\mathrm{Na}_{2} \mathrm{O}$ by the $5 \mathrm{CaO} .3 \mathrm{Al}_{2} \mathrm{O}_{3}$, as reported by Brownmiller and Bogue [5]. However, such an explanation is not necessarily the true one, as the mere presence of $\mathrm{Na}_{2} \mathrm{O}$ as a fourth component may be sufficient to cause both $2 \mathrm{CaO} \cdot \mathrm{Al}_{2} \mathrm{O}_{3} \cdot \mathrm{SiO}_{2}$ and $5 \mathrm{CaO} \cdot 3 \mathrm{Al}_{2} \mathrm{O}_{3}$ to be mutually stable in the presence of liquid.

The primary phase crystallizing in the region labeled $2 \mathrm{Na}_{2} \mathrm{O} .3 \mathrm{CaO} .5 \mathrm{Al}_{2} \mathrm{O}_{3}$ in figure 3 has optical properties corresponding to those reported for this end member of the solid solution series $\mathrm{Na}_{2} \mathrm{O} \cdot \mathrm{Al}_{2} \mathrm{O}_{3}-2 \mathrm{Na}_{2} \mathrm{O} .3 \mathrm{CaO} .5 \mathrm{Al}_{2} \mathrm{O}_{3}$ in the ternary system $\mathrm{Na}_{2} \mathrm{O}-\mathrm{CaO}-$ $\mathrm{Al}_{2} \mathrm{O}_{3}$ [5]. If the phase contains $\mathrm{Na}_{2} \mathrm{O} \cdot \mathrm{Al}_{2} \mathrm{O}_{3}$, it was not detectable through observation of the optical properties.

The fields of $3 \mathrm{CaO} .5 \mathrm{Al}_{2} \mathrm{O}_{3}$ and $\mathrm{Al}_{2} \mathrm{O}_{3}$ were determined only approximately as they are far removed from the compositions of chief interest in this work. However, it was observed that the $3 \mathrm{CaO} .5 \mathrm{Al}_{2} \mathrm{O}_{3}$ crystallizing in this plane corresponds to the "stable" form of Rankin and Wright [4], and that the alumina was in the form of corundum, or $\alpha \mathrm{Al}_{2} \mathrm{O}_{3}$.

\section{THE PLANE $2 \mathrm{CaO} . \mathrm{SiO}_{2}-\mathrm{CaO}-\left(\mathrm{Na}_{2} \mathrm{O}+3 \mathrm{Al}_{2} \mathrm{O}_{3}\right)$}

Figure 4 shows the equilibrium diagram for this plane. It is constructed from the data of table 2. A number of marked changes in the primary phase regions have occurred in comparison to the preceding plane. Eight crystalline phases are indicated on the diagram, as follows: $\mathrm{CaO}, 3 \mathrm{CaO} . \mathrm{SiO}_{2}, 2 \mathrm{CaO} . \mathrm{SiO}_{2}, \mathrm{Na}_{2} \mathrm{O} .8 \mathrm{CaO} .3 \mathrm{Al}_{2} \mathrm{O}_{3}, 5 \mathrm{CaO} .3-$ $\mathrm{Al}_{2} \mathrm{O}_{3}, \quad 2 \mathrm{CaO} . \mathrm{Al}_{2} \mathrm{O}_{3} \cdot \mathrm{SiO}_{2}, \quad 2 \mathrm{Na}_{2} \mathrm{O} .3 \mathrm{CaO} .5 \mathrm{Al}_{2} \mathrm{O}_{3}$ solid solution, and $\beta-\mathrm{Al}_{2} \mathrm{O}_{3}$.

The composition of the compound $\mathrm{Na}_{2} \mathrm{O} .8 \mathrm{CaO} .3 \mathrm{Al}_{2} \mathrm{O}_{3}$ is located on the $\mathrm{CaO}-\left(\mathrm{Na}_{2} \mathrm{O}+3 \mathrm{Al}_{2} \mathrm{O}_{3}\right)$ side, but as this phase melts incongruently to $\mathrm{CaO}$ and liquid, it is in the $\mathrm{CaO}$ primary phase area. The field of $\mathrm{Na}_{2} \mathrm{O} .8 \mathrm{CaO} .3 \mathrm{Al}_{2} \mathrm{O}_{3}$ is displaced away from the $\mathrm{CaO}$ apex, and extends from the $\mathrm{CaO}-\left(\mathrm{Na}_{2} \mathrm{O}+3 \mathrm{Al}_{2} \mathrm{O}_{3}\right)$ side toward the $2 \mathrm{CaO} \cdot \mathrm{SiO}_{2}$ apex. It may be observed that $\mathrm{Na}_{2} \mathrm{O} .8 \mathrm{CaO} .3 \mathrm{Al}_{2} \mathrm{O}_{3}$ exists in equilibrium with $2 \mathrm{CaO} . \mathrm{SiO}_{2}$ and liquids whose compositions lie on the curve FG. The 
experimental results indicate that the $3 \mathrm{CaO} . \mathrm{SiO}_{2}$ field narrows to a point not indicated in figure 4 , slightly to the left of point $\mathrm{F}$, since $3 \mathrm{CaO} . \mathrm{SiO}_{2}$ and $\mathrm{Na}_{2} \mathrm{O} .8 \mathrm{CaO} .3 \mathrm{Al}_{2} \mathrm{O}_{3}$ were not observed to exist together in equilibrium with liquid in any of the quenched charges. It is probable, therefore, that $3 \mathrm{CaO} . \mathrm{SiO}_{2}$ crystallizing from compositions in this plane decomposes upon cooling into $2 \mathrm{CaO} . \mathrm{SiO}_{2}$ and $\mathrm{CaO}$ just before the crystallization of $\mathrm{Na}_{2} \mathrm{O} .8 \mathrm{CaO} .3 \mathrm{Al}_{2} \mathrm{O}_{3}$ begins.

It follows from the near meeting of the fields for the four compounds $\mathrm{CaO}, 3 \mathrm{CaO} . \mathrm{SiO}_{2}, 2 \mathrm{CaO} . \mathrm{SiO}_{2}$, and $\mathrm{Na}_{2} \mathrm{O} .8 \mathrm{CaO} .3 \mathrm{Al}_{2} \mathrm{O}_{3}$, that an invariant point for these phases and liquid must be located near point $\mathrm{F}$, but at a slightly smaller $\mathrm{Na}_{2} \mathrm{O} / \mathrm{Al}_{2} \mathrm{O}_{3}$ ratio. Point $\mathrm{F}$ has a composition very close to that of mix 107 , table 2 , and the invariant point, which will be referred to as point 3 , is estimated to have the following composition: 5.0 percent $\mathrm{Na}_{2} \mathrm{O}, 54.1$ percent $\mathrm{CaO}, 28.6$ percent $\mathrm{Al}_{2} \mathrm{O}_{3}$, and 12.3 percent $\mathrm{SiO}_{2}$. It has a temperature estimated to be $1,445^{\circ} \pm$ $10^{\circ} \mathrm{C}$, based on data for mixes 105 and 107 . Point 3 is not a eutectic.

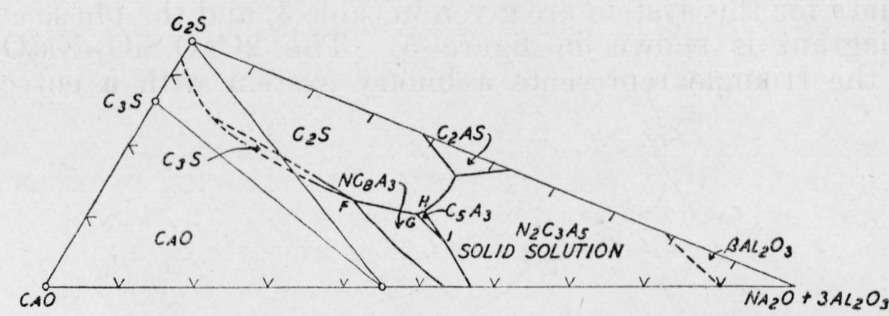

Figure 4.-Composition plane $2 \mathrm{CaO} . \mathrm{SiO}_{2}-\mathrm{CaO}-\left(\mathrm{Na}_{2} \mathrm{O}+3 \mathrm{Al}_{2} \mathrm{O}_{3}\right)$.

It will be noted that the primary phase area of $5 \mathrm{CaO} .3 \mathrm{Al}_{2} \mathrm{O}_{3}$ is very small, and that if the plane of the diagram be imagined to swing gradually toward higher $\mathrm{Na}_{2} \mathrm{O}_{/} \mathrm{Al}_{2} \mathrm{O}_{3}$ ratios, the points $\mathrm{G}, \mathrm{H}$, and $\mathrm{I}$ would converge on a single point which represents the invariant point for the phases $2 \mathrm{CaO} . \mathrm{SiO}_{2}, \mathrm{Na}_{2} \mathrm{O} .8 \mathrm{CaO} .3 \mathrm{Al}_{2} \mathrm{O}_{3}, 5 \mathrm{CaO} 3 \mathrm{Al}_{2} \mathrm{O}_{3}$, $2 \mathrm{Na}_{2} \mathrm{O} .3 \mathrm{CaO} .5 \mathrm{Al}_{2} \mathrm{O}_{3}$ solid solution, and liquid. It is designated as point 5, and has the following estimated composition: 8.0 percent $\mathrm{Na}_{2} \mathrm{O}, 46.1$ percent $\mathrm{CaO}, 35.5$ percent $\mathrm{Al}_{2} \mathrm{O}_{3}$, and 10.4 percent $\mathrm{SiO}_{2}$. Point 5 must be a eutectic as it lies within the composition tetrahedron formed by $2 \mathrm{CaO} . \mathrm{SiO}_{2}, \quad \mathrm{Na}_{2} \mathrm{O} .8 \mathrm{CaO} .3 \mathrm{Al}_{2} \mathrm{O}_{3}, \quad 5 \mathrm{CaO} .3 \mathrm{Al}_{2} \mathrm{O}_{3}$, and $2 \mathrm{Na}_{2} \mathrm{O} .3 \mathrm{CaO} .5 \mathrm{Al}_{2} \mathrm{O}_{3}$. It is a eutectic, regardless of the amount of $\mathrm{Na}_{2} \mathrm{O} \cdot \mathrm{Al}_{2} \mathrm{O}_{3}$ in solid solution in $2 \mathrm{Na}_{2} \mathrm{O} .3 \mathrm{CaO} .5 \mathrm{Al}_{2} \mathrm{O}_{3}$. The temperature of point 5 , based on data for mix 93 , is estimated to be $1,340^{\circ}$ $\pm 10^{\circ} \mathrm{C}$.

The composition of point 5 lies close to the $2 \mathrm{CaO} . \mathrm{SiO}_{2}-$ $\mathrm{Na}_{2} \mathrm{O} .8 \mathrm{CaO} .3 \mathrm{Al}_{2} \mathrm{O}_{3}-2 \mathrm{Na}_{2} \mathrm{O} .3 \mathrm{CaO} .5 \mathrm{Al}_{2} \mathrm{O}_{3}$ join, which means that the percentage of $5 \mathrm{CaO} .3 \mathrm{Al}_{2} \mathrm{O}_{3}$ at complete crystallization will be very small, provided that the compounds are pure. On this basis the phase composition was calculated to be 29.8 percent $2 \mathrm{CaO} . \mathrm{SiO}_{2}, 34.8$ percent $\mathrm{Na}_{2} \mathrm{O} .8 \mathrm{CaO} .3 \mathrm{Al}_{2} \mathrm{O}_{3}, 34.8$ percent $2 \mathrm{Na}_{2} \mathrm{O} .3 \mathrm{CaO} .5 \mathrm{Al}_{2} \mathrm{O}_{3}$ and 0.6 percent $5 \mathrm{CaO} .3 \mathrm{Al}_{2} \mathrm{O}_{3}$. However, it is known that the $5 \mathrm{CaO} .3 \mathrm{Al}_{2} \mathrm{O}_{3}$ contains about 1 percent $\mathrm{Na}_{2} \mathrm{O}$ in solid solution, which would increase the $5 \mathrm{CaO} .3 \mathrm{Al}_{2} \mathrm{O}_{3}$ to 1.0 percent. Moreover, if the $2 \mathrm{Na}_{2} \mathrm{O} .3 \mathrm{CaO} .5 \mathrm{Al}_{2} \mathrm{O}_{3}$ in equilibrium at the eutectic temperature contains $\mathrm{Na}_{2} \mathrm{O} \cdot \mathrm{Al}_{2} \mathrm{O}_{3}$ in solid solution, as is indicated by a lowering of the mean index of refraction from 1.592 to about 1.587 , the proportion of $5 \mathrm{CaO}^{2} \mathrm{Al}_{2} \mathrm{O}_{3}$ will be 
further increased. Since the actual amount of this latter solid solution is not known, it is not possible to calculate the exact phase composition resulting from complete crystallization of a melt of the composition of point 5 .

The compound $3 \mathrm{CaO} .5 \mathrm{Al}_{2} \mathrm{O}_{3}$ was not observed as a primary phase in the plane $2 \mathrm{CaO} \cdot \mathrm{SiO}_{2}-\mathrm{CaO}-\left(\mathrm{Na}_{2} \mathrm{O}+3 \mathrm{Al}_{2} \mathrm{O}_{3}\right)$. However, the form of $3 \mathrm{CaO} .5 \mathrm{Al}_{2} \mathrm{O}_{3}$ termed "unstable" by Rankin and Wright [4] occurs as a secondary phase in mix 56, and as a tertiary phase in mix 57. It appears to be in a state of stable equilibrium in these charges. The crystals have an index of refraction of about 1.67 and a low double refraction.

The form of $\mathrm{Al}_{2} \mathrm{O}_{3}$ which crystallizes from mixtures in this plane is $\beta \mathrm{Al}_{2} \mathrm{O}_{3}$, which appears to be in stable equilibrium. The optical properties agree with those reported by Brownmiller and Bogue [5].

\section{THE SYSTEM $2 \mathrm{CaO} \cdot \mathrm{SiO}_{2} \mathrm{CaO}-\mathrm{Na}_{2} \mathrm{O} \cdot \mathrm{Al}_{2} \mathrm{O}_{3}$}

The data for this system are given in table 3 , and the phase equilibrium diagram is shown in figure 5. The $2 \mathrm{CaO} \cdot \mathrm{SiO}_{2}-\mathrm{Na}_{2} \mathrm{O} \cdot \mathrm{Al}_{2} \mathrm{O}_{3}$ side of the triangle represents a binary system with a eutectic at

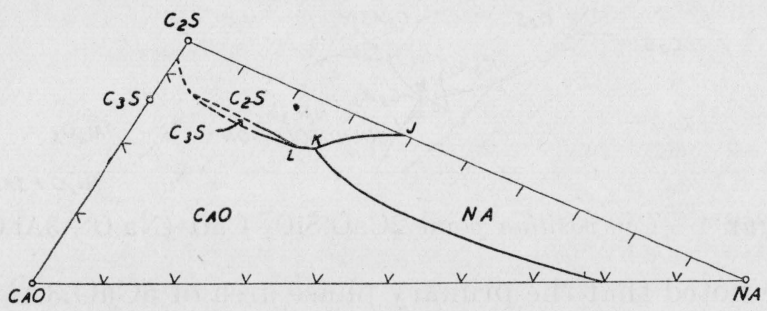

Figure 5.-System $2 \mathrm{CaO} . \mathrm{SiO}_{2}-\mathrm{CaO}-\mathrm{Na}_{2} \mathrm{O} \cdot \mathrm{Al}_{2} \mathrm{O}_{3}$.

61 percent $2 \mathrm{CaO} . \mathrm{SiO}_{2}$ and 39 percent $\mathrm{Na}_{2} \mathrm{O} . \mathrm{Al}_{2} \mathrm{O}_{3}$, or on an oxide basis, 14.7 percent $\mathrm{Na}_{2} \mathrm{O}, 39.7$ percent $\mathrm{CaO}, 24.3$ percent $\mathrm{Al}_{2} \mathrm{O}_{3}$, and 21.3 percent $\mathrm{SiO}_{2}$. This eutectic has a temperature of $1,405^{\circ}$ $\pm 10^{\circ} \mathrm{C}$ and is indicated by the letter $\mathrm{J}$ in figure 5 .

Except for the existence of a small amount of solid solution in the $2 \mathrm{CaO} . \mathrm{SiO}_{2}$, and probably also in the $\mathrm{Na}_{2} \mathrm{O} \cdot \mathrm{Al}_{2} \mathrm{O}_{3}$, the system $2 \mathrm{CaO} . \mathrm{SiO}_{2}$ $\mathrm{CaO}-\mathrm{Na}_{2} \mathrm{O} \cdot \mathrm{Al}_{2} \mathrm{O}_{3}$ is ternary. Due to experimental difficulties the phase relations as determined are not considered to be as accurate as those obtained with mixture of lower $\mathrm{Na}_{2} \mathrm{O} / \mathrm{Al}_{2} \mathrm{O}_{3}$ ratios. Not only is the rate of volatilization of $\mathrm{Na}_{2} \mathrm{O}$ higher, but the time required for the attainment of equilibrium, particularly in charges containing crystalline $3 \mathrm{CaO} \cdot \mathrm{SiO}_{2}$, is much increased. This compound was found to form extremely slowly by reaction of $2 \mathrm{CaO} \cdot \mathrm{SiO}_{2}$ and $\mathrm{CaO}$. Increasing the temperature does not appreciably accelerate the combination as the $\mathrm{CaO}$ tends to form large, unreactive crystals. Consequently, for charges where $3 \mathrm{CaO} . \mathrm{SiO}_{2}$ was expected as a stable phase, the original mixes were prepared with $3 \mathrm{CaO} . \mathrm{SiO}_{2}$ instead of $2 \mathrm{CaO} . \mathrm{SiO}_{2}$ and $\mathrm{CaO}$. In this way it is believed that a nearer approach to equilibrium was attained.

The results indicate that the phase equilibrium relations are in general as shown in figure 5 . Point $\mathrm{K}$ is a ternary eutectic for $2 \mathrm{CaO}$. $\mathrm{SiO}_{2}, \mathrm{CaO}, \mathrm{Na}_{2} \mathrm{O} \cdot \mathrm{Al}_{2} \mathrm{O}_{3}$, and liquid. It occurs at a compound composition of approximately 56 percent $2 \mathrm{CaO} . \mathrm{SiO}_{2}, 16$ percent $\mathrm{CaO}$, 
and 28 percent $\mathrm{Na}_{2} \mathrm{O} \cdot \mathrm{Al}_{2} \mathrm{O}_{3}$, or in terms of oxides, 11 percent $\mathrm{Na}_{2} \mathrm{O}$, 52 percent $\mathrm{CaO}, 17$ percent $\mathrm{Al}_{2} \mathrm{O}_{3}$, and 20 percent $\mathrm{SiO}_{2}$. The temperature, based on data for mix 33 , table 3 , is $1,355 \pm 10^{\circ} \mathrm{C}$.

It will be noticed that the primary field of $3 \mathrm{CaO} . \mathrm{SiO}_{2}$ in figure 5 is drawn as narrowing to a point at $\mathrm{L}$. The basis for this is the fact that $3 \mathrm{CaO} . \mathrm{SiO}_{2}$ was never observed in charges containing crystalline $\mathrm{Na}_{2} \mathrm{O} \cdot \mathrm{Al}_{2} \mathrm{O}_{3}$. The solid phases in equilibrium with $\mathrm{Na}_{2} \mathrm{O} \cdot \mathrm{Al}_{2} \mathrm{O}_{3}$ were always $2 \mathrm{CaO} . \mathrm{SiO}_{2}$ and $\mathrm{CaO}$. Accordingly, point $\mathrm{L}$ represents an invariant point for $3 \mathrm{CaO} . \mathrm{SiO}_{2}, 2 \mathrm{CaO} . \mathrm{SiO}_{2}, \mathrm{CaO}$, and liquid, and indicates a lower temperature limit of stability for $3 \mathrm{CaO} . \mathrm{SiO}_{2}$ in this system. Thus $3 \mathrm{CaO} . \mathrm{SiO}_{2}$ should decompose into $2 \mathrm{CaO} . \mathrm{SiO}_{2}$ and $\mathrm{CaO}$ when the temperature is lowered below that of point $\mathrm{L}$. A partial decomposition of $3 \mathrm{CaO} . \mathrm{SiO}_{2}$ was observed for several charges in the system, but attempts to locate the temperature of point $\mathrm{L}$ were not successful because of the slowness of the reaction. When once formed, $3 \mathrm{CaO} \cdot \mathrm{SiO}_{2}$ tends to persist in the melt, although held at constant temperature for many hours. According to the phase diagram, point $\mathrm{L}$ should have a temperature above $1,355^{\circ} \mathrm{C}$.

This behavior of $3 \mathrm{CaO} . \mathrm{SiO}_{2}$ is interesting in the light of the work of Carlson [11], who found that $3 \mathrm{CaO} . \mathrm{SiO}_{2}$ tends to dissociate into $2 \mathrm{CaO} \cdot \mathrm{SiO}_{2}$ and $\mathrm{CaO}$ when heated at temperatures between $1,000^{\circ}$ and $1,300^{\circ} \mathrm{C}$. The possibility was pointed out by Jänecke [12] that with a suitable third component, a ternary system with $\mathrm{CaO}$ and $2 \mathrm{CaO} . \mathrm{SiO}_{2}$ might be obtained in which the field of $3 \mathrm{CaO} . \mathrm{SiO}_{2}$ narrows to a point at both ends, meeting the boundary curve between $\mathrm{CaO}$ and $2 \mathrm{CaO} \cdot \mathrm{SiO}_{2}$ at an invariant point. It is interesting to note that Eitel has since reported that such a situation exists in the system $2 \mathrm{CaO} . \mathrm{SiO}_{2}-\mathrm{CaO}-\mathrm{CaF}_{2}$ [13]. It appears from the present work that $\mathrm{Na}_{2} \mathrm{O} \cdot \mathrm{Al}_{2} \mathrm{O}_{3}$ also is such a component. The theoretical considerations involved in a limited temperature range of stability for $3 \mathrm{CaO} . \mathrm{SiO}_{2}$ are discussed by Lea and Parker [7].

The $2 \mathrm{CaO} . \mathrm{SiO}_{2}$ phase which was observed in the binary system $2 \mathrm{CaO} . \mathrm{SiO}_{2}-\mathrm{Na}_{2} \mathrm{O} . \mathrm{Al}_{2} \mathrm{O}_{3}$ has the optical properties of the $\beta$ form. This was confirmed by X-ray diffraction patterns. ${ }^{2}$ If there is any solid solution in the $\beta-2 \mathrm{CaO} . \mathrm{SiO}_{2}$ it must be very small. However, in several compositions in the interior of the system the $2 \mathrm{CaO} . \mathrm{SiO}_{2}$ phase corresponded in properties to one of the $\alpha-2 \mathrm{CaO} . \mathrm{SiO}_{2}$ solid solutions described in a previous paper [6]. The extent of this solid solution was not determined and the relation which it may bear to the stability of the $3 \mathrm{CaO} . \mathrm{SiO}_{2}$ has not been investigated. A somewhat similar situation with regard to solid solution appears to exist for the $\mathrm{Na}_{2} \mathrm{O} \cdot \mathrm{Al}_{2} \mathrm{O}_{3}$. Along the join $2 \mathrm{CaO} \cdot \mathrm{SiO}_{2}-\mathrm{Na}_{2} \mathrm{O} . \mathrm{Al}_{2} \mathrm{O}_{3}$ the properties agree with those of the pure compound, but in compositions containing added $\mathrm{CaO}$ a slight increase in the indices of refraction indicate that the phase may be one in the solid solution series $\mathrm{Na}_{2} \mathrm{O} \cdot \mathrm{Al}_{2} \mathrm{O}_{3}$ $2 \mathrm{Na}_{2} \mathrm{O} .3 \mathrm{CaO} .5 \mathrm{Al}_{2} \mathrm{O}_{3}$.

\section{ADDITIONAL COMPOSITIONS LOCATED BETWEEN THE $2 \mathrm{CaO} \mathrm{SiO}_{2}$ - $\mathrm{CaO}-\left(\mathrm{Na}_{2} \mathrm{O}+6 \mathrm{Al}_{2} \mathrm{O}_{3}\right)$ AND $2 \mathrm{CaO} \cdot \mathrm{SiO}_{2}-\mathrm{CaO}-\left(\mathrm{Na}_{2} \mathrm{O}+3 \mathrm{Al}_{2} \mathrm{O}_{3}\right)$ PLANES}

To locate invariant point 1 at which $2 \mathrm{CaO}^{-\mathrm{SiO}_{2}}, 3 \mathrm{CaO} . \mathrm{SiO}_{2}$, $3 \mathrm{CaO} . \mathrm{Al}_{2} \mathrm{O}_{3}$, and $\mathrm{Na}_{2} \mathrm{O} .8 \mathrm{CaO} .3 \mathrm{Al}_{2} \mathrm{O}_{3}$ are in equilibrium with liquid, it became necessary to investigate a number of mixtures not included in

${ }^{2}$ All X-ray patterns were made by H. F. McMurdie. 
the original composition planes. The additional data are given in table 4. They show that mix 206 is approximately the composition of point 1. The phases, $2 \mathrm{CaO} . \mathrm{SiO}_{2}, 3 \mathrm{CaO} . \mathrm{SiO}_{2}, 3 \mathrm{CaO} . \mathrm{Al}_{2} \mathrm{O}_{3}$, $\mathrm{Na}_{2} \mathrm{O} .8 \mathrm{CaO} .3 \mathrm{Al}_{2} \mathrm{O}_{3}$, and glass, were identified in a charge of mix 206 quenched at $1,440^{\circ} \mathrm{C}$. The composition is therefore placed at 3.5 percent $\mathrm{Na}_{2} \mathrm{O}, 55.2$ percent $\mathrm{CaO}, 31.0$ percent $\mathrm{Al}_{2} \mathrm{O}_{3}$, and 10.3 percent $\mathrm{SiO}_{2}$, and the temperature at $1,440^{\circ} \pm 10^{\circ} \mathrm{C}$. Point 1 is not a eutectic, and therefore a mixture of this composition does not become completely crystalline on cooling when $1,440^{\circ}$ is reached, but continues to contain liquid down to $1,340^{\circ}$, the temperature of point 5 , the only quaternary eutectic in this part of the system.

One other quaternary invariant point, not a eutectic, is located near point 1. This is the point for $\mathrm{CaO}, 3 \mathrm{CaO} . \mathrm{SiO}_{2}, 3 \mathrm{CaO} \cdot \mathrm{Al}_{2} \mathrm{O}_{3}$, $\mathrm{Na}_{2} \mathrm{O} .8 \mathrm{CaO} .3 \mathrm{Al}_{2} \mathrm{O}_{3}$, and liquid. Using figures 3 and 4 , it is estimated by interpolation that this point, referred to as point 2 , has a composition only slightly higher in $\mathrm{CaO}$ and lower in $\mathrm{SiO}_{2}$ than point 1 , or approximately 3.5 percent $\mathrm{Na}_{2} \mathrm{O}, 56.0$ percent $\mathrm{CaO}, 31.0$ percent $\mathrm{Al}_{2} \mathrm{O}_{3}$, and 9.5 percent $\mathrm{SiO}_{2}$. According to theory, point 2 must have a higher temperature than point 1, but it can be only slightly higher, since both points must have temperatures below that of point 3 , which has been determined as $1,445^{\circ} \pm 10^{\circ} \mathrm{C}$. To indicate this relationship point 2 is accordingly given the temperature $1,442^{\circ} \pm 10^{\circ} \mathrm{C}$.

\section{DISCUSSION}

\section{CRYSTALLIZATION IN THE SYSTEM}

For purposes of discussion and comparison, the data obtained for the five quaternary invariant points determined in this investigation are grouped together in table 5. Of these only point 5 is a eutectic. The others are reaction points, and melts of these compositions upon complete equlibrium crystallization consist of sets of solid phases differing from those in equilibrium with liquid at the respective invariant points. A melt of the composition of point 2 when cooled under equilibrium conditions becomes completely crystalline at $1,365^{\circ} \mathrm{C}$, the temperature of point 4 , and consists of $2 \mathrm{CaO} \cdot \mathrm{SiO}_{2}$, $3 \mathrm{CaO} . \mathrm{Al}_{2} \mathrm{O}_{3}, 5 \mathrm{CaO} .3 \mathrm{Al}_{2} \mathrm{O}_{3}$, and $\mathrm{Na}_{2} \mathrm{O} .8 \mathrm{CaO} .3 \mathrm{Al}_{2} \mathrm{O}_{3}$. Melts having compositions of points 1,3 , and 4 become completely crystalline at $1,340^{\circ} \mathrm{C}$, the temperature of point 5 , and consist of $2 \mathrm{CaO} \cdot \mathrm{SiO}_{2}$, $5 \mathrm{CaO} .3 \mathrm{Al}_{2} \mathrm{O}_{3}, \quad \mathrm{Na}_{2} \mathrm{O} .8 \mathrm{CaO} .3 \mathrm{Al}_{2} \mathrm{O}_{3}$, and $2 \mathrm{Na}_{2} \mathrm{O} .3 \mathrm{CaO} .5 \mathrm{Al}_{2} \mathrm{O}_{3}$ solid solution.

The existence of invariant point 1 shows that the compounds $2 \mathrm{CaO} . \mathrm{SiO}_{2}, 3 \mathrm{CaO} \cdot \mathrm{SiO}_{2}, 3 \mathrm{CaO} \cdot \mathrm{Al}_{2} \mathrm{O}_{3}$, and $\mathrm{Na}_{2} \mathrm{O} .8 \mathrm{CaO} .3 \mathrm{Al}_{2} \mathrm{O}_{3}$ may be final products of crystallization in mixtures of $\mathrm{Na}_{2} \mathrm{O}, \mathrm{CaO}, \mathrm{Al}_{2} \mathrm{O}_{3}$, and $\mathrm{SiO}_{2}$ approaching in composition that of portland cement clinker. Such mixtures are located in the tetrahedron formed by the compositions of the four solid phases in equilibrium at point 1 . When these mixtures are heated, the first stable liquid is produced at $1,440^{\circ} \mathrm{C}$, and this liquid has the composition of point 1 . Conversely, when melts in this tetrahedron are cooled under equilibrium conditions, the last liquid (of the composition of point 1) disappears at $1,440^{\circ} \mathrm{C}$. 
TABLE 5.-Quaternary invariant points in the system $\mathrm{Na}_{2} \mathrm{O}-\mathrm{CaO}-\mathrm{Al}_{2} \mathrm{O}_{3}-\mathrm{SiO}_{2}$

\begin{tabular}{|c|c|c|c|c|c|c|}
\hline \multirow{2}{*}{ Point } & \multirow{2}{*}{$\begin{array}{c}\text { Temper- } \\
\text { ature }\end{array}$} & \multirow{2}{*}{ Solid phases } & \multicolumn{4}{|c|}{ Composition } \\
\hline & & & $\mathrm{Na}_{2} \mathrm{O}$ & $\mathrm{CaO}$ & $\mathrm{Al}_{2} \mathrm{O}_{3}$ & $\mathrm{SiO}_{2}$ \\
\hline $\begin{array}{l}1 \\
2 \\
3 \\
4 \\
5 \\
5\end{array}$ & $\begin{array}{r}\circ \\
C \pm 10^{\circ} \\
1,440 \\
1,442 \\
1,445 \\
1,365 \\
1,340\end{array}$ & $\begin{array}{l}\mathrm{C}_{2} \mathrm{~S}, \mathrm{C}_{3} \mathrm{~S}, \mathrm{C}_{3} \mathrm{~A}, \mathrm{NC}_{8} \mathrm{~A}_{3} \\
\mathrm{CaO}, \mathrm{C}_{3} \mathrm{~S}, \mathrm{C}_{3} \mathrm{~A}, \mathrm{NC}_{8} \mathrm{~A}_{3} \\
\mathrm{CaO}, \mathrm{C}_{3} \mathrm{~S}, \mathrm{C}_{2} \mathrm{~S}, \mathrm{NC}_{8} \mathrm{~A}_{3} \\
\mathrm{C}_{2} \mathrm{~S}, \mathrm{C}_{3} \mathrm{~A}, \mathrm{C}_{5} \mathrm{~A}_{3}, \mathrm{NC}_{8} \mathrm{~N}_{3} \\
\mathrm{C}_{2} \mathrm{~S}, \mathrm{C}_{5} \mathrm{~A}_{3}, \mathrm{NC}_{8} \mathrm{~A}_{3}, \mathrm{~N}_{2} \mathrm{C}_{3} \mathrm{~A}_{5}\end{array}$ & $\begin{array}{r}\% \\
3.5 \\
3.5 \\
5.0 \\
3.5 \\
8.0\end{array}$ & $\begin{array}{l}\% \\
55.2 \\
56.0 \\
54.1 \\
50.2 \\
46.1\end{array}$ & $\begin{array}{l}\% \\
31.0 \\
31.0 \\
28.6 \\
37.6 \\
35.5\end{array}$ & $\begin{array}{r}\% \\
10.3 \\
9.5 \\
12.3 \\
8.7 \\
10.4\end{array}$ \\
\hline
\end{tabular}

The various possible courses of crystallization are many and complex for mixtures in the $2 \mathrm{CaO} . \mathrm{SiO}_{2}-3 \mathrm{CaO} . \mathrm{SiO}_{2}-3 \mathrm{CaO} . \mathrm{Al}_{2} \mathrm{O}_{3}-\mathrm{Na}_{2} \mathrm{O}$.$8 \mathrm{CaO} .3 \mathrm{Al}_{2} \mathrm{O}_{3}$ tetrahedron. However, the liquids of all such compositions eventually arrive at point 1 as the melt is cooled. In some melts the liquid composition passes through point 2 before point 1 is reached. $3 \mathrm{CaO} \cdot \mathrm{Al}_{2} \mathrm{O}_{3}$ and $\mathrm{Na}_{2} \mathrm{O} \cdot 8 \mathrm{CaO} \cdot 3 \mathrm{Al}_{2} \mathrm{O}_{3}$ do not crystallize out together until either point 2 or point 1 is reached. In melts whose course of crystallization passes through point $2,3 \mathrm{CaO} \cdot \mathrm{Al}_{2} \mathrm{O}_{3}$ appears before $\mathrm{Na}_{2} \mathrm{O} .8 \mathrm{CaO} .3 \mathrm{Al}_{2} \mathrm{O}_{3}$ when the $\mathrm{Na}_{2} \mathrm{O} / \mathrm{Al}_{2} \mathrm{O}_{3}$ ratio of the original mixture is less than that of point 2 . Likewise, in melts whose course of crystallization does not pass through point 2 , but arrives at point 1 directly, $3 \mathrm{CaO} \cdot \mathrm{Al}_{2} \mathrm{O}_{3}$ crystallizes before $\mathrm{Na}_{2} \mathrm{O} .8 \mathrm{CaO} .3 \mathrm{Al}_{2} \mathrm{O}_{3}$ when the $\mathrm{Na}_{2} \mathrm{O} / \mathrm{Al}_{2} \mathrm{O}_{3}$ ratio of the original mixture is less than that of point 1 . Since points 1 and 2 have the same $\mathrm{Na}_{2} \mathrm{O} / \mathrm{Al}_{2} \mathrm{O}_{3}$ ratio within experimental error, this ratio determines whether $3 \mathrm{CaO} . \mathrm{Al}_{2} \mathrm{O}_{3}$ or $\mathrm{Na}_{2} \mathrm{O} .8 \mathrm{CaO}$.$3 \mathrm{Al}_{2} \mathrm{O}_{3}$ is first to crystallize on cooling the melts under discussion. On a weight basis, the $\mathrm{Na}_{2} \mathrm{O} / \mathrm{Al}_{2} \mathrm{O}_{3}$ ratio of points 1 and 2 is 0.11 . Therefore in mixtures which have $\mathrm{Na}_{2} \mathrm{O} / \mathrm{Al}_{2} \mathrm{O}_{3}$ ratios less than 0.11 , $3 \mathrm{CaO} \cdot \mathrm{Al}_{2} \mathrm{O}_{3}$ appears before $\mathrm{Na}_{2} \mathrm{O} .8 \mathrm{CaO} .3 \mathrm{Al}_{2} \mathrm{O}_{3}$, whereas in those which have $\mathrm{Na}_{2} \mathrm{O} / \mathrm{Al}_{2} \mathrm{O}_{3}$ ratios greater than $0.11, \mathrm{Na}_{2} \mathrm{O} .8 \mathrm{CaO} .3 \mathrm{Al}_{2} \mathrm{O}_{3}$ precedes $3 \mathrm{CaO} . \mathrm{Al}_{2} \mathrm{O}_{3}$.

\section{SOLID SOLUTIONS}

Although it has been demonstrated by the present work that $3 \mathrm{CaO} . \mathrm{Al}_{2} \mathrm{O}_{3}$ and $\mathrm{Na}_{2} \mathrm{O} .8 \mathrm{CaO} .3 \mathrm{Al}_{2} \mathrm{O}_{3}$ may exist together as distinct phases in stable equilibrium with liquid at the invariant point where $2 \mathrm{CaO} . \mathrm{SiO}_{2}$ and $3 \mathrm{CaO} . \mathrm{SiO}_{2}$ are also present, the data thus far reported do not give any information concerning the relation between these compounds below the temperature of liquid formation. Previous data of this laboratory have indicated that, with slow cooling or prolonged heating at lower temperatures, $3 \mathrm{CaO} \cdot \mathrm{Al}_{2} \mathrm{O}_{3}$ and $\mathrm{Na}_{2} \mathrm{O} .8 \mathrm{CaO} .3 \mathrm{Al}_{2} \mathrm{O}_{3}$ form a complete solid solution series in the ternary system $\mathrm{Na}_{2} \mathrm{O}$ $\mathrm{CaO}-\mathrm{Al}_{2} \mathrm{O}_{3}$ [14]. In order to test the possibility of the formation of similar solid solutions in the quaternary system, a mix was made up which was calculated to have the following potential compound composition: 39.9 percent $3 \mathrm{CaO} . \mathrm{Al}_{2} \mathrm{O}_{3}, 40.1$ percent $\mathrm{Na}_{2} \mathrm{O} .8 \mathrm{CaO} .3 \mathrm{Al}_{2} \mathrm{O}_{3}$, and 20.0 percent $2 \mathrm{CaO} . \mathrm{SiO}_{2}$. A sample of this mixture was given two 30 -minute heatings at $1,425^{\circ} \mathrm{C}$, with intermediate grinding, and was examined microscopically and a portion taken for X-ray analysis.

Under the microscope the charge appeared to consist of a large amount of material having a mottled gray interference color, along with a small amount of $2 \mathrm{CaO} \cdot \mathrm{SiO}_{2}$. No glass was detected. The double refraction of the predominant material was appreciably less 
than that of $\mathrm{Na}_{2} \mathrm{O} \cdot 8 \mathrm{CaO} \cdot 3 \mathrm{Al}_{2} \mathrm{O}_{3}$. No distinct grains of either $3 \mathrm{CaO}$.$\mathrm{Al}_{2} \mathrm{O}_{3}$ or $\mathrm{Na}_{2} \mathrm{O} .8 \mathrm{CaO} .3 \mathrm{Al}_{2} \mathrm{O}_{3}$ could be identified. Although the double refraction did not appear to be entirely uniform, there was definite evidence that a reaction had taken place to produce a phase or phases that were neither pure $3 \mathrm{CaO} . \mathrm{Al}_{2} \mathrm{O}_{3}$ nor pure $\mathrm{Na}_{2} \mathrm{O} .8 \mathrm{CaO} .3 \mathrm{Al}_{2} \mathrm{O}_{3}$.

Unfortunately, the powder diffraction patterns of both $3 \mathrm{CaO} \cdot \mathrm{Al}_{2} \mathrm{O}_{3}$ and $\mathrm{Na}_{2} \mathrm{O} .8 \mathrm{CaO} \cdot 3 \mathrm{Al}_{2} \mathrm{O}_{3}$ are very similar. By comparing the data of McMurdie [15] for $3 \mathrm{CaO} \cdot \mathrm{Al}_{2} \mathrm{O}_{3}$ with that of Brownmiller and Bogue [5] for $\mathrm{Na}_{2} \mathrm{O} .8 \mathrm{CaO} .3 \mathrm{Al}_{2} \mathrm{O}_{3}$, only minor differences are seen. The pattern for the mixture of $3 \mathrm{CaO} . \mathrm{Al}_{2} \mathrm{O}_{3}, \mathrm{Na}_{2} \mathrm{O} .8 \mathrm{CaO} .3 \mathrm{Al}_{2} \mathrm{O}_{3}$ and $2 \mathrm{CaO} . \mathrm{SiO}_{2}$ mentioned in a previous paragraph, is also very similar, but resembles that of $\mathrm{Na}_{2} \mathrm{O} .8 \mathrm{CaO} .3 \mathrm{Al}_{2} \mathrm{O}_{3}$ more than that of $3 \mathrm{CaO} \cdot \mathrm{Al}_{2} \mathrm{O}_{3}$. It contains a number of pairs of lines located close together. Such pairs occur also with $\mathrm{Na}_{2} \mathrm{O} .8 \mathrm{CaO} .3 \mathrm{Al}_{2} \mathrm{O}_{3}$, but to a lesser extent, while in the pattern of $3 \mathrm{CaO} . \mathrm{Al}_{2} \mathrm{O}_{3}$ only single lines occur. In table 6 are shown the interplanar spacings calculated for the charge prepared from the mixture, in comparison to the more important lines of $3 \mathrm{CaO} \cdot \mathrm{Al}_{2} \mathrm{O}_{3}$ and $\mathrm{Na}_{2} \mathrm{O} .8 \mathrm{CaO} .3 \mathrm{Al}_{2} \mathrm{O}_{3}$.

TABLE 6.-Powder diffraction patterns of $3 \mathrm{CaO} . \mathrm{Al}_{2} \mathrm{O}_{3}, \mathrm{Na}_{2} \mathrm{O} .8 \mathrm{CaO} .3 \mathrm{Al}_{2} \mathrm{O}_{3}$, and a mixture of $3 \mathrm{CaO} . \mathrm{Al}_{2} \mathrm{O}_{3}, \mathrm{Na}_{2} \mathrm{O} .8 \mathrm{CaO} .3 \mathrm{Al}_{2} \mathrm{O}_{3}$, and $2 \mathrm{CaO}^{-\mathrm{SiO}_{2}}$ after heating to $1,425^{\circ} C$

[VS=very strong, $\mathrm{S}=$ strong, $\mathrm{M}=$ medium, $\mathrm{W}=$ weak, $\mathrm{VW}=$ very weak]

\begin{tabular}{|c|c|c|c|c|c|}
\hline \multicolumn{2}{|c|}{$3 \mathrm{CaO} \cdot \mathrm{Al}_{2} \mathrm{O}_{3}$ (McMurdie) } & \multicolumn{2}{|c|}{$\begin{array}{c}3 \mathrm{CaO} \cdot \mathrm{Al}_{2} \mathrm{O}_{3}-\mathrm{Na}_{2} \mathrm{O} .8 \mathrm{CaO} \\
3 \mathrm{Al}_{2} \mathrm{O}_{3}-2 \mathrm{CaO} \cdot \mathrm{SiO}_{2} \text { mixture }\end{array}$} & \multicolumn{2}{|c|}{$\begin{array}{c}\mathrm{Na}_{2} \mathrm{O} .8 \mathrm{CaO} .3 \mathrm{Al}_{2} \mathrm{O}_{3} \\
\text { (Brownmiller and Bogue) }\end{array}$} \\
\hline $\mathrm{d} h k l$ & $\begin{array}{l}\text { Estimated } \\
\text { intensity }\end{array}$ & $\mathrm{d} h k l$ & $\begin{array}{l}\text { Estimated } \\
\text { intensity }\end{array}$ & $\mathrm{d} h k l$ & $\begin{array}{l}\text { Estimated } \\
\text { intensity }\end{array}$ \\
\hline 3.34 & vW & 3.41 & $\mathrm{~W}$ & 3.37 & W \\
\hline 2.70 & vs & $\begin{array}{l}3.33 \\
2.69\end{array}$ & $\begin{array}{l}\text { VW } \\
\text { VS }\end{array}$ & 2.68 & VS \\
\hline 2.39 & W & $\begin{array}{l}2.42 \\
2.37\end{array}$ & $\begin{array}{l}\mathrm{W} \\
\mathrm{W}\end{array}$ & $\begin{array}{l}2.41 \\
2.37\end{array}$ & $\begin{array}{l}\text { W } \\
W\end{array}$ \\
\hline 2. 258 & VW & 2.27 & VW & & \\
\hline 2. 200 & $\mathrm{M}$ & 2. 196 & $\mathrm{M}$ & 2. 20 & $\mathrm{~S}$ \\
\hline $\begin{array}{l}2.039 \\
1.984\end{array}$ & $\begin{array}{l}\text { VW } \\
\text { VW }\end{array}$ & & W & 2.03 & W \\
\hline $\begin{array}{l}1.951 \\
1.907\end{array}$ & $\begin{array}{c}\mathrm{VW} \\
\mathrm{s}\end{array}$ & $\begin{array}{l}1.958 \\
1.918\end{array}$ & $\begin{array}{l}\text { VW } \\
\text { S }\end{array}$ & 1.915 & $\mathrm{~S}$ \\
\hline 1.826 & VW & $\begin{array}{l}1.888 \\
1.745\end{array}$ & $\begin{array}{c}\mathrm{M} \\
\mathrm{VW}\end{array}$ & $\begin{array}{l}1.888 \\
1.746\end{array}$ & $\stackrel{\mathrm{S}}{\mathrm{W}}$ \\
\hline 1. 556 & $\mathrm{~S}$ & 1. 564 & $\stackrel{\mathrm{S}}{\mathrm{M}}$ & 1.558 & VS \\
\hline 1. 346 & M & 1. 356 & W & 1. 347 & $\mathrm{M}$ \\
\hline 1.206 & M & $\begin{array}{l}1.346 \\
1.213\end{array}$ & $M$ & 1.210 & M \\
\hline $\begin{array}{l}1.106 \\
1.023\end{array}$ & $\begin{array}{l}\mathrm{W} \\
\mathrm{M}\end{array}$ & $\begin{array}{l}1.197 \\
1.102 \\
1.023\end{array}$ & $\begin{array}{l}\text { W } \\
W \\
M\end{array}$ & $\begin{array}{l}\text { 1. } 103 \\
1.019\end{array}$ & $\mathrm{~W}$ \\
\hline & & $\begin{array}{l}1.025 \\
1.015\end{array}$ & $\mathrm{M}$ & & \\
\hline
\end{tabular}

${ }^{1}$ Lines corresponding to those of $2 \mathrm{CaO} . \mathrm{SiO}_{2}$ are omitted. , Very broad.

These microscopical and X-ray data are limited, but it seems justifiable to conclude that at temperatures below that of liquid formation in the system $\mathrm{Na}_{2} \mathrm{O}-\mathrm{CaO}-\mathrm{Al}_{2} \mathrm{O}_{3}-\mathrm{SiO}_{2}, 3 \mathrm{CaO} \cdot \mathrm{Al}_{2} \mathrm{O}_{3}$, and $\mathrm{Na}_{2} \mathrm{O} .8 \mathrm{CaO} .3 \mathrm{Al}_{2} \mathrm{O}_{3}$ may combine under suitable conditions to form a solid solution phase having intermediate optical properties and a crystal structure similar to that of $\mathrm{Na}_{2} \mathrm{O} .8 \mathrm{CaO} .3 \mathrm{Al}_{2} \mathrm{O}_{3}$.

In the majority of the compositions studied in this investigation the $2 \mathrm{CaO} . \mathrm{SiO}_{2}$ possessed optical properties corresponding to those of the $\beta$ form. In a few cases, however, as in the sytem $2 \mathrm{CaO} . \mathrm{SiO}_{2}-$ 
CaO- $\mathrm{Na}_{2} \mathrm{O} \cdot \mathrm{Al}_{2} \mathrm{O}_{3}$, the $2 \mathrm{CaO} . \mathrm{SiO}_{2}$ appeared to be one of the $\alpha-2 \mathrm{CaO} \cdot \mathrm{SiO}_{2}$ solid solutions described in a previous paper [6]. No definite region of occurrence for this phase in contrast to $\beta-2 \mathrm{CaO} . \mathrm{SiO}_{2}$ was observed, and the amounts of $\mathrm{Na}_{2} \mathrm{O}$ and $\mathrm{Al}_{2} \mathrm{O}_{3}$ in solid solutions were not determined. Consequently in the tables and diagrams the two forms of $2 \mathrm{CaO} . \mathrm{SiO}_{2}$ are not differentiated.

Measurements of indices of refraction have indicated that solid solution of $\mathrm{Na}_{2} \mathrm{O}$ and $\mathrm{Al}_{2} \mathrm{O}_{3}$ may occur to a limited extent in $\beta-2 \mathrm{CaO} . \mathrm{SiO}_{2}$ [6]. There is evidence that such solid solution may also take place in the ternary system $\mathrm{Na}_{2} \mathrm{O}-\mathrm{CaO}-\mathrm{SiO}_{2}$. Preliminary experiments with compositions along the join $2 \mathrm{CaO} . \mathrm{SiO}_{2}-\mathrm{Na}_{2} \mathrm{O}$.$\mathrm{CaO} . \mathrm{SiO}_{2}$ have given apparently a single phase up to a molar ratio of $1 \mathrm{Na}_{2} \mathrm{O} \cdot \mathrm{CaO} \cdot \mathrm{SiO}_{2}$ to $142 \mathrm{CaO} \cdot \mathrm{SiO}_{2}$. It was at first thought that this might represent a new ternary compound of the approximate formula $\mathrm{Na}_{2} \mathrm{O} .29 \mathrm{CaO} .15 \mathrm{SiO}_{2}$. It has a complexly twinned structure very similar to that of the $\mathrm{K}_{2} \mathrm{O} .23 \mathrm{CaO} .12 \mathrm{SiO}_{2}$ phase described by Taylor [16]. However, the powder diffraction patterns obtained for the $\mathrm{Na}_{2} \mathrm{O}$ phase have always corresponded to that of $\beta-2 \mathrm{CaO} \cdot \mathrm{SiO}_{2}$, with a slight shift of some of the lines. Consequently, it must be concluded that it is not a distinct compound but rather a solid solution phase. As mentioned in the previous paper [6], it is probable that the twinning results from the inversion of the $2 \mathrm{CaO} . \mathrm{SiO}_{2}$ from the $\alpha$ to the $\beta$ form.

\section{APPLICATIONS TO PORTLAND CEMENT CLINKER}

The results of this investigation are limited in their application to commercial portland cement by the fact that one of the major components of clinker, $\mathrm{Fe}_{2} \mathrm{O}_{3}$, has been excluded. Furthermore, the exact effect of a number of the minor components is not yet known. Nevertheless, on the basis of present information, it is possible to reach a much more definite conclusion than heretofore regarding the state of the $\mathrm{Na}_{2} \mathrm{O}$ in clinker. A number of possibilities are indicated, depending not only on the composition of the clinker, but perhaps even more on the heat treatment which it receives.

If cooling has been rapid so that glass is present in the clinker, some of the $\mathrm{Na}_{2} \mathrm{O}$ will always be found in this glass. The percentage of $\mathrm{Na}_{2} \mathrm{O}$ occurring in this manner will depend upon individual conditions, but some indication may be obtained from the phase equilibrium data for the $\mathrm{Na}_{2} \mathrm{O}-\mathrm{CaO}-\mathrm{Al}_{2} \mathrm{O}_{3}-\mathrm{SiO}_{2}$ system. For example, a glass resulting from the quenching of a liquid near in composition to that of point 1 contains approximately 3.5 percent $\mathrm{Na}_{2} \mathrm{O}$. However, if this same melt is allowed to crystallize independently until the composition of the remaining liquid approaches that of point 5 , and then is quenched, the resulting glass will contain upward of 8 percent $\mathrm{Na}_{2} \mathrm{O}$.

The results thus far obtained indicate that when the cooling takes place at a slower rate, some of the $\mathrm{Na}_{2} \mathrm{O}$ in a clinker may crystallize as the compound $\mathrm{Na}_{2} \mathrm{O} .8 \mathrm{CaO} \cdot 3 \mathrm{Al}_{2} \mathrm{O}_{3}$, or more likely as a solid solution phase of this compound and $3 \mathrm{CaO} \cdot \mathrm{Al}_{2} \mathrm{O}_{3}$. It is probable that this phase is closely related to the "prismatic dark interstitical" material found in some commercial portland cement clinkers. The compound, $\mathrm{Na}_{2} \mathrm{O} .8 \mathrm{CaO} .3 \mathrm{Al}_{2} \mathrm{O}_{3}$ has been observed in the present work to form prismatic crystals when the cooling rate was suitable, and Taylor [17] has shown that the dark prismatic phase of clinker is closely related to $680952-46-7$ 
$3 \mathrm{CaO} \cdot \mathrm{Al}_{2} \mathrm{O}_{3}$. Although he found no proportionality between the percentage of alkali in a clinker and the amount of the dark prismatic phase which may be produced, the presence of either $\mathrm{Na}_{2} \mathrm{O}$ or $\mathrm{K}_{2} \mathrm{O}$ appears to be necessary for its formation.

The fact that $2 \mathrm{CaO} \cdot \mathrm{SiO}_{2}$ can form solid solutions containing $\mathrm{Na}_{2} \mathrm{O}$ makes this another possible manner of occurrence in clinker. The opinion was expressed in a previous paper [6] that because of their instability, the $\alpha-2 \mathrm{CaO} . \mathrm{SiO}_{2}$ solid solutions which may be formed at clinkering temperatures, probably are not present in normaly cooled clinker. However, since the material held in solid solution at the higher temperature is precipitated as inclusions in the $2 \mathrm{CaO} . \mathrm{SiO}_{2}$ grains when they invert to the $\beta$ form, some of the $\mathrm{Na}_{2} \mathrm{O}$ will be contained in these inclusions. This fact may be of importance in the hydration of cement, since $\beta-2 \mathrm{CaO} . \mathrm{SiO}_{2}$ hydrates slowly and the alkali imbedded in the grains might be protected from the action of the water for considerable lengths of time.

Addition of $\mathrm{Na}_{2} \mathrm{O}$ to the system $\mathrm{CaO}-\mathrm{Al}_{2} \mathrm{O}_{3}-\mathrm{SiO}_{2}$ was found to result in an enlargement of the primary phase region of $\mathrm{CaO}$. This is evident from an examination of figures $2,3,4$, and 5 . This is important since it means that certain compositions in the alkali-free system would not contain free $\mathrm{CaO}$ at clinkering temperatures, but after addition of $\mathrm{Na}_{2} \mathrm{O}$ would contain stable $\mathrm{CaO}$. In this way $\mathrm{Na}_{2} \mathrm{O}$ can affect adversely the combination of the $\mathrm{CaO}$ in the cement mix.

\section{SUMMARY}

The portion of the quaternary system $\mathrm{Na}_{2} \mathrm{O}-\mathrm{CaO}-\mathrm{Al}_{2} \mathrm{O}_{3}-\mathrm{SiO}_{2}$ of importance to portland cement technology has been studied by the quenching method. The phase equilibrium data are presented in the form of tables and phase diagrams, and features of interest are discussed.

Mixtures located in three definite composition planes passing through the four-component tetrahedron, as well as a number of additional compositions, were investigated. A total of 12 crystalline compounds were observed to have primary phase volumes within this portion of the system.

Five of the quaternary invariant points occurring in this part of the system have been located approximately. Of these only one is a eutectic; the other four are reaction points. It was found that $2 \mathrm{CaO} . \mathrm{SiO}_{2}, 3 \mathrm{CaO} . \mathrm{SiO}_{2}, 3 \mathrm{CaO} . \mathrm{Al}_{2} \mathrm{O}_{3}$, and $\mathrm{Na}_{2} \mathrm{O} .8 \mathrm{CaO} .3 \mathrm{Al}_{2} \mathrm{O}_{3}$ are capable of coexisting with liquid at one of these reaction points. The temperature of this point is $1,440^{\circ} \pm 10^{\circ} \mathrm{C}$ and the composition is 3.5 percent $\mathrm{Na}_{2} \mathrm{O}$, 55.2 percent $\mathrm{CaO}, 31.0$ percent $\mathrm{Al}_{2} \mathrm{O}_{3}$, and 10.3 percent $\mathrm{SiO}_{2}$.

Evidence was obtained indicating that at temperatures below that of liquid formation, $3 \mathrm{CaO} . \mathrm{Al}_{2} \mathrm{O}_{3}$ and $\mathrm{Na}_{2} \mathrm{O} .8 \mathrm{CaO} .3 \mathrm{Al}_{2} \mathrm{O}_{3}$ may combine under suitable conditions to form a solid solution phase of intermediate properties and crystal structure.

Based on present information, and necessarily ignoring the effect of $\mathrm{Fe}_{2} \mathrm{O}_{3}$ and minor components, it is concluded that the $\mathrm{Na}_{2} \mathrm{O}$ in portland cement clinker may occur in several possible ways, depending on individual conditions:

1 . As a component of glass. 
2. As $\mathrm{Na}_{2} \mathrm{O} .8 \mathrm{CaO} .3 \mathrm{Al}_{2} \mathrm{O}_{3}$ or a solid solution of that compound with $3 \mathrm{CaO} \cdot \mathrm{Al}_{2} \mathrm{O}_{3}$. Such a phase may be associated with the prismatic dark interstitial material frequent]y observed in clinker.

3. As a solid solution of a soda-bearing phase in $2 \mathrm{CaO} \cdot \mathrm{SiO}_{2}$.

4. As inclusions of a soda-bearing phase in $\beta-2 \mathrm{CaO} . \mathrm{SiO}_{2}$ produced by ex-solution on inversion from $\alpha-2 \mathrm{CaO} \cdot \mathrm{SiO}_{2}$.

\section{REFERENCES}

[1] W. C. Hansen, J. Am. Chem. Soc. 50, 3081 (1928).

[2] H. F. McMurdie and H. Insley, J. Research NBS 16, 467 (1936) RP884.

[3] H. Insley and H. F. McMurdie, J. Research NBS 20, 173 (1938) RP1074.

[4] G. A. Rankin and F. E. Wright, Am. J. Sci. [4] 39, 1 (1915).

[5] L. T. Brownmiller and R. H. Bogue, BS J. Research 8, 289 (1932) RP414; Am. J. Sci. [5] 23, 501 (1932).

[6] K. T. Greene, J. Research NBS 32, 1 (1944) RP1570.

[7] F. M. Lea and T. W. Parker, Trans. Roy. Soc. (London) [A] 234, 1 (1934).

[8] J. F. Schairer, J. Am. Ceram. Soc. 25, 241 (1942).

[9] L. T. Brownmiller, Am. J. Sci. [5] 29, 260 (1935).

[10] W. C. Taylor, J. Research NBS 21, 315 (1938) RP1131.

[11] E. T. Carlson, BS J. Research \%, 893 (1931) RP381.

[12] E. Jänecke, Zement 21, 377 (1932).

[13] W. Eitel, Physicalische Chemie der Silikate, 2d ed., p. 735, (J. A. Barth, Leipzig, 1941).

[14] R. H. Bogue, Symposium on the Chemistry of Cements, p. 65 (Royal Sweedish Institute for Engineering Research, Stockholm, 1938).

[15] H. F. McMurdie, J. Research NBS 27, 499 (1941) RP1437.

[16] W. C. Taylor, J. Research NBS 27, 311 (1941) RP1421.

[17] W. C. Taylor, J. Research NBS 30, 329 (1943) RP1536.

[18] W. Bussem and A. Eitel, Z. Krist. [A] 95, 175 (1936).

[19] K. Lagerquist, A. Wallmark and A. Westgren, Z. anorg. allgem. Chem. 234, 1 (1937).

[20] W. Bussem, Symposium on the Chemistry of Cements, p. 149 (Stockholm, 1938).

[21] T. Thorvaldson and W. G. Schneider, Canadian J. Research 19, 109 (1941).

Washington, October 18, 1945. 